\title{
ERGODIC COCYCLES OF IDPFT SYSTEMS AND NONSINGULAR GAUSSIAN ACTIONS
}

\author{
AleXANDRe I. DANilenko AND Mariusz LemańCZyK \\ To the memory of Sergiy Sinel'shchikov, our colleague and friend
}

\begin{abstract}
It is proved that each Gaussian cocycle over a mildly mixing Gaussian transformation is either a Gaussian coboundary or sharply weak mixing. The class of nonsingular infinite direct products $T$ of transformations $T_{n}, n \in \mathbb{N}$, of finite type (IDPFT) is studied. It is shown that if $T_{n}$ is mildly mixing, $n \in \mathbb{N}$, the sequence of the Radon-Nikodym derivatives of $T_{n}$ is asymptotically translation quasi-invariant and $T$ is conservative then the Maharam extension of $T$ is sharply weak mixing. This techniques provides a new approach to the nonsingular Gaussian transformations studied recently by Arano, Isono and Marrakchi.
\end{abstract}

\section{INTRODUCTION}

The original motivation of this paper was to tackle a problem (stated in [LeLeSk]) that is related to the theory of Gaussian dynamical systems: let $T$ be an ergodic (equivalently, weakly mixing) Gaussian transformation on a standard probability space $(X, \mathfrak{B}, \mu)$ and let $H$ be the corresponding invariant Gaussian subspace of the real Hilbert space $L_{0}^{2}(X, \mu)$.

Conjecture. For each function $f \in H$, either $f$ is a T-coboundary (equivalently, a Gaussian coboundary) or the skew product transformation $T_{f}$ acting on $X \times \mathbb{R}$ is ergodic.

In this paper we obtain the affirmative answer under a slightly stronger assumption than the weak mixing. We say that a nonsingular transformation $R$ is sharply weak mixing if the direct product of $R$ with each ergodic conservative transformation is either totally dissipative or ergodic. In particular, $R$ is ergodic. We also recall that $T_{f}$ is conservative for each $f \in H$.

Theorem 0.1. If $T$ is mildly mixing and $f$ is not a coboundary for $T$ then $T_{f}$ is sharply weak mixing.

To prove Theorem 0.1 we note that there exists a decomposition of $T$ into direct product of mildly mixing transformations $T_{n}$ in a such a way that $f$ splits into a sum of coboundaries $f_{n}:=a_{n}-a_{n} \circ T_{n}$ for $T_{n}, n \in \mathbb{N}$. Moreover, the sequence of

The main results of the paper were obtained during the visit of the first named author at Nicolaus Copernicus University in March-April 2020. The staying was supported by a special research grant of NCU.

Research of the second named author was supported by Narodowe Centrum Nauki grant UMO2019/33/B/ST1/00364. 
distributions of the transfer functions $\left(a_{n}\right)_{n=1}^{\infty}$ satisfies a certain property that we call ATI (asymptotic translation invariance) in Definition 1.2. Then Theorem 0.1 follows from the next theorem.

Theorem 0.2. Given a locally compact second countable Abelian group $G$, a sequence of mildly mixing dynamical systems $\left(X_{n}, \nu_{n}, T_{n}\right)$ and a sequence of functions $f_{n}: X_{n} \rightarrow G, n \in \mathbb{N}$, consider the infinite direct product $(X, \nu, T):=$ $\bigotimes_{n=1}^{\infty}\left(X_{n}, \nu_{n}, T_{n}\right)$. Suppose that a function $f(x):=\sum_{n=1}^{\infty}\left(f_{n}\left(T_{n} x_{x}\right)-f_{n}\left(x_{n}\right)\right) \in G$ is well defined for $\nu$-a.e. $x=\left(x_{n}\right)_{n=1}^{\infty} \in X$. If the sequence of distributions $\left(\nu_{n} \circ f_{n}^{-1}\right)_{n \in \mathbb{N}}$ on $G$ is ATI and the $f$-skew product extension $T_{f}: X \times G \rightarrow X \times G$ of $T$ is conservative then $T_{f}$ is sharply weak mixing.

The proof of Theorem 0.2 is based on the two ideas:

- the mild mixing and the product structure of $T_{f}$ yield that each $T_{f}$-invariant subset is also invariant under a large group of "finitary" transformations, i.e. transformations that "move" finitely many coordinates only;

- the ATI property implies that this finitary group is ergodic via techniques related to computation of the essential values of cocycles.

The first aforementioned idea was inspired by the proof [ArIsMa, Theorem D] on ergodic properties of some nonsingular Gaussian group actions.

We then turn to classical problems of nonsingular ergodic theory. We mention a recent progress in providing natural examples for nonsingular ergodic theory: nonsingular Bernoulli and Markov shiftwise actions (see [DaLe], [KoSo], [Av], [MaVa] and references therein), nonsingular Gaussian systems [ArIsMa], nonsingular Poisson systems ([DaKoRo1], [DaKoRo2]). In the present work we introduce one more natural family of nonsingular transformations. We say that a nonsingular transformation $T$ on a standard probability space $(X, \mu)$ is an infinite direct product of finite types (IDPFT) if there is a sequence of ergodic probability preserving dynamical systems $\left(X_{n}, \nu_{n}, T_{n}\right)$ and a sequence of probability measures $\mu_{n}$ on $X_{n}, n \in \mathbb{N}$, such that $\mu_{n} \sim \nu_{n}$ for each $n$ and $(X, \mu, T)=\bigotimes_{n=1}^{\infty}\left(X_{n}, \mu_{n}, T_{n}\right)$. Kakutani's theorem [Ka] provides a criterion where $\mu$ is quasi-invariant under $T$. We are interested in the case where $\mu \perp \nu$ and $\mu$ does not admit an equivalent $T$-invariant probability. It is possible that $(X, \mu, T)$ is totally dissipative. Moreover, we show that for each ergodic conservative nonsingular transformation $S$, the product $T \times S$ is either totally dissipative or conservative.

Theorem 0.3. Let $\left(X_{n}, \nu_{n}, T_{n}\right)$ be mildly mixing for each $n>0$. If $T$ is $\mu$ conservative and the sequence of distributions of the random variables $\log \frac{d \mu_{n}}{d \nu_{n}}, n \in$ $\mathbb{N}$, is ATQI then $T$ is ergodic of stable type Krieger's type III $I_{1}$. Moreover, the Maharam extension of $T$ is sharply weak mixing.

The property ATQI (asymptotically translation quasi-invariantness, see Definition 2.8) in the statement of Theorem 0.3 is an analogue of ATI though neither ATI implies ATQI nor vice-versa. The scheme of the proof of Theorem 0.3 is similar to the that of Theorem 0.2 and we use again the aforementioned two ideas. However, there is a "nonsingular" nuance. Namely, a formal repetition of the proof of Theorem 0.2 yields that the group of finitary transformations is ergodic with respect to the "wrong" measure. Hence, it does not work. We recall that there are two different (mutually singular) natural measures associated with a IDPFT system: $\nu$ (invariant) and $\mu$ (quasiinvariant). Therefore a certain additional argument and 
the property ATQI instead of ATI are needed to prove ergodicity for the "right" measure. We also provide examples of rigid IDPFT systems $T$ of Krieger's type $I I I_{\lambda}$ for an arbitrary $\lambda \in(0,1)$.

We have already mentioned that the nonsingular Gaussian systems were studied recently in [ArIsMa]. However, the exposition there is based heavily on the affine geometry and often use a nonstandard (from the dynamical viewpoint) terminology. Therefore, we decided to provide here an alternative exposition of this important topic. We define the nonsingular Gaussian systems as transformations on Hilbert spaces $\mathcal{H}$ furnished with Gaussian measures stressing on the fact that the systems are compositions of classical Guassian automorphisms and totally dissipative transformations (given by nonsingular rotations). Connections with the underlying Fock space, the first chaos and the exponential map are enlighten explicitly. We also explain interrelation between the nonsingular Gaussian systems and the nonsingular Poisson systems. Our main observation is that the Gaussian transformations (out of a "small" family of degenerated ones) is a subclass of IDPFT systems. Hence we deduce from Theorem 0.2 one of the main results of [ArIsMa] $\left(\mathcal{H}_{0}\right.$ below is a linear subspace of $\mathcal{H}$ endowed with a new inner product, see Section 3$)$.

Theorem 0.4. Let an orthogonal operator $V$ of a real Hilbert space $\mathcal{H}_{0}$ be mildly mixing. Let $f \in \mathcal{H}_{0}$ not be a $V$-coboundary, i.e. $f \neq V a-a$ for any $a \in \mathcal{H}_{0}$. If the nonsingular Gaussian transformation $T_{(f, V)}$ associated with the pair $(f, V)$ is conservative then the Maharam extension of $T_{(f, V)}$ is sharply weak mixing. In particular, $T_{(f, V)}$ is of type $I I I_{1}$.

The outline of the paper is as follows. In Section 1 we introduce main definitions: Hellinger distance, weak mixing properties for nonsingular actions, ATI property, skew product extension, essential value of a cocycle, etc. Then we prove Theorem 0.2 (see Theorem 1.5) and deduce Theorem 0.1 from it (see Theorem 1.6). We also provide a generalization of Theorem 0.1 (see Conjecture II and a discussion above it). In Section 2 we consider nonsingular versions of the problems studied in $\S 1$. IDPFT systems are introduced in Definition 2.2. Radon-Nikodym cocycle, Maharam extension and Kriger's types $I I I_{\lambda}, 0 \leq \lambda \leq 1$, are discussed there. We show that each IDPFT system is either conservative or totally dissipative (Corollary 2.7), introduce the ATQI property (Definition 2.8) and prove Theorem 0.3 (Theorem 2.10). Type $I I I_{\lambda}$ rigid IDPFT systems are also constructed there for each $\lambda \in(0.1)$ (Proposition 2.12). The final Section 3 is devoted to nonsingular Gaussian systems. We first recall the definition of Gaussian measure in a separable Hilbert space. Then we discuss the main properties of the related Fock space and exponential map. Given an orthogonal operator $V$ in a Hilbert space $\mathcal{H}_{0}$ and a vector $f \in \mathcal{H}_{0}$, we associate a nonsingular transformation $T_{(f, V)}$ acting on the corresponding Hilbert space $\mathcal{H} \supset \mathcal{H}_{0}$ equipped with a Gaussian measure $\mu$. We show that $T_{(f, V)}$ is the composition of the classic Gaussian $\mu$-preserving transformation associated to $V$ with the (totally dissipative) rotation by $f$. It is well known that the nonsingular transformation group $\left\{T_{(f, 0)} \mid f \in \mathcal{H}_{0}\right\}$ generated by the rotations is ergodic (see, e.g. [Gu]) but the Kriger's type has not been specified so far. We prove that it is $I I I_{1}$ (Theorem 3.7). We show that the Koopman operator generated by $T_{(f, V)}$ is the Weyl operator associated to the pair $(f / 2, V)$. A criterion for the existence of an invariant equivalent probability measure for $T_{(f, V)}$ is established in Theorem 3.9 (cf. [DAKoRo1, Proposition 6.4] and [ArIsMa]). Theorem 0.4 in proved in this section (Theorem 3.12). 
After completion of this paper we learnt about a work [MaVa] devoted to nonsingular Gaussian actions of arbitrary groups. It was written independently but simultaneously with our work. ${ }^{1}$ Some of our results overlap with theirs. Say, Theorem 3.7 is [MaVa, Theorem 3.1] and Theorem 0.1, though stated in a more general form, is, in fact, equivalent to [MaVa, Theorem 9.1(3)] in case of $\mathbb{Z}$-actions. Our proofs are different. They are based solely on elementary techniques of the nonsingular ergodic (measurable orbit) theory. We do not use affine geometry, representation theory nor harmonic analysis.

\section{WeAK MiXing COCYCles of PRODUCt type.}

1.1. Hellinger distance and Kakutani's theorem. Let $\gamma$ and $\delta$ be two equivalent probability measures on a standard Borel space $(Y, \mathfrak{C})$. The square of the Hellinger distance between $\gamma$ and $\delta$ is

$$
H^{2}(\gamma, \delta):=\frac{1}{2} \int_{Y}\left(1-\sqrt{\frac{d \gamma}{d \delta}}\right)^{2} d \delta=1-\int_{Y} \sqrt{\frac{d \gamma}{d \delta}} d \delta
$$

By the Cauchy-Schwarz inequality, $0 \leq H(\gamma, \delta)<1$. We also remind [Ni] the following inequalities between the Hellinger distance and the total variation:

$$
H^{2}(\gamma, \delta) \leq\|\gamma-\delta\|_{1}:=\sup _{C \in \mathfrak{C}}|\gamma(C)-\delta(C)| \leq \sqrt{2} H(\gamma, \delta)
$$

We now state the Kakutani theorem on equivalence of infinite products of probability measures $[\mathrm{Ka}]$.

Theorem A. Let $\mu_{n}$ and $\nu_{n}$ be two equivalent probability measures on a standard Borel space $\left(X_{n}, \mathfrak{B}_{n}\right)$ for each $n \in \mathbb{N}$. Let $\mu$ and $\nu$ denote the infinite product measures $\bigotimes_{n \in \mathbb{N}} \mu_{n}$ and $\bigotimes_{n \in \mathbb{N}} \nu_{n}$ respectively on the standard Borel space $(X, \mathfrak{B}):=$ $\bigotimes_{n \in \mathbb{N}}\left(X_{n}, \mathfrak{B}_{n}\right)$. If

$$
\prod_{n=1}^{\infty}\left(1-H^{2}\left(\mu_{n}, \nu_{n}\right)\right)>0 \text { or, equivalently, } \sum_{n=1}^{\infty} H^{2}\left(\mu_{n}, \nu_{n}\right)<\infty
$$

then $\mu \sim \nu, \prod_{n=1}^{\infty}\left(1-H^{2}\left(\mu_{n}, \nu_{n}\right)\right)=1-H^{2}(\mu, \nu)$ and $\frac{d \mu}{d \nu}(x)=\prod_{n \in \mathbb{N}} \frac{d \mu_{n}}{d \nu_{n}}\left(x_{n}\right)$ at a.e. $x=\left(x_{n}\right)_{n \in \mathbb{N}} \in X$. If (1-2) does not hold then $\mu \perp \nu$.

1.2. Weak mixing properties of nonsingular actions. We recall that given a non-singular transformation $R$ of a standard Borel probability space $(Y, \mathfrak{C}, \nu)$, there is a unique decomposition $Y=\mathcal{D}(R) \sqcup \mathcal{C}(R)$ (called Hopf's decomposition) of $Y$ into two Borel sets such that $\mathcal{D}(R)$ is the disjoint union of the orbit of a wandering set $W$, i.e. $\mathcal{D}(R)=\bigsqcup_{n \in \mathbb{Z}} R^{n} W$ and $\mathcal{C}(R)=Y \backslash \mathcal{D}(R)$ contains no non-trivial wandering set. If $\mathcal{C}(R)=Y$ then $R$ is called conservative and if $\mathcal{D}(R)=Y$ then $R$ is called totally dissipative. As both parts $\mathcal{C}(R)$ and $\mathcal{D}(R)$ are $R$-invariant, each ergodic $R$ is either conservative or totally dissipative. An ergodic conservative nonsingular transformation $R$ is called weakly mixing if for each ergodic probability preserving transformation $S$, the Cartesian product $R \times S$ is ergodic. We now introduce a stronger concept of weak mixing.

\footnotetext{
${ }^{1}$ The two papers appeared on ArXiv in two successive days. We thank S. Vaes for informing us about $[\mathrm{MaVa}]$.
} 
Definition 1.1. An ergodic conservative nonsingular transformation $R$ is called sharply weak mixing if for each ergodic conservative nonsingular transformation $S$, the direct product $R \times S$ is either totally dissipative or ergodic.

If $S$ in the above definition admits an equivalent invariant probability measure (i.e. $S$ is of type $I I_{1}$ ) then $T \times S$ is conservative (see [Aa, Proposition 1.1.6, part 2]). Hence $R \times S$ is ergodic according to Definition 1.1. Thus, every sharply weak mixing transformation is weakly mixing. It follows from [SiTh] that every conservative nonsingular transformation with property $K$ is sharply weak mixing (see also [AaLiWe, Theorem 6.7] for other examples). In [AdFrSi] and [Da] examples of weakly mixing infinite measure preserving rank-one transformations $R$ were constructed such that $R \times R$ is conservative but not ergodic. Hence $R$ is not sharply weak mixing. We recall that an ergodic probability preserving transformation $R$ defined on a space $(Y, \mathfrak{C}, \nu)$ is called mildly mixing ([FuWe], see also [AaLiWe] and [ScWa] $)$ if every function $f \in L^{\infty}(\nu)$ such that $\left\|f \circ T^{n_{i}}-f\right\|_{1} \rightarrow 0$ for some sequence $n_{i} \rightarrow \infty$ is constant.

We will utilize the following result from $[\mathrm{ScWa}]$.

Theorem B. Let $R$ be a mildly mixing transformation of a standard probability space $(Y, \mathfrak{C}, \nu)$ and let $C$ be a conservative nonsingular transformation of a standard probability space $(Z, \mathfrak{F}, \tau)$. If a function $F \in L^{\infty}(Y \times Z, \nu \otimes \tau)$ is invariant under $R \times C$ then there is $f \in L^{\infty}(Z, \tau)$ such that $F(y, z)=f(z)$ a.e.

We note that Theorem $\mathrm{B}$ was proved in [ScWa] for the ergodic conservative $C$ only but the proof remains valid for an arbitrary conservative $C$ as well. Direct products of finitely (and countably) many mildly mixing transformatations are mildly mixing.

It follows from Theorem B that an ergodic finite measure preserving transformation is sharply weak mixing if and only if it is mildly mixing. In Theorems 1.5 and 1.6 below we will provide examples of mildly mixing transformations (including zero entropy case) which have locally compact group extensions that are sharply weak mixing infinite measure preserving (and hence not mildly mixing).

1.3. ATI property. Fix a locally compact second countable Abelian group $G$. Denote by $\lambda_{G}$ a Haar measure on $G$.

Definition 1.2. A sequence $\left(\xi_{n}\right)_{n=1}^{\infty}$ of probability Borel measures on $G$ is called asymptotically translation invariant (ATI) if

$$
\lim _{m \rightarrow \infty}\left\|\xi_{n} * \xi_{n+1} * \cdots * \xi_{n+m} * \delta_{a}-\xi_{n} * \xi_{n+1} * \cdots * \xi_{n+m}\right\|_{1}=0
$$

for each $n \in \mathbb{N}$ and $a \in G$.

Example 1.3. Let $\mathcal{N}_{a, \sigma^{2}}$ denote the normal distribution on $\mathbb{R}$ with parameters $a$ and $\sigma^{2}$, i.e. $\widehat{\mathcal{N}_{a, \sigma^{2}}}(t)=e^{i a t-\frac{1}{2} \sigma^{2} t^{2}}$ for all $t \in \mathbb{R}$. Given two sequences $\left(a_{n}\right)_{n \in \mathbb{Z}}$ and $\left(\sigma_{n}\right)_{n=1}^{\infty}$ of reals such that $\sum_{n=1}^{\infty} \sigma_{n}^{2}=+\infty$, the sequence of probabilities $\left(\mathcal{N}_{a_{n}, \sigma_{n}^{2}}\right)_{n=1}^{\infty}$ is ATI. Indeed,

$$
\begin{aligned}
\mathcal{N}_{a_{n}, \sigma_{n}^{2}} * \cdots * \mathcal{N}_{a_{n+m}, \sigma_{n+m}^{2}} & =\mathcal{N}_{\sum_{k=n}^{n+m} a_{k}, \sum_{k=n}^{n+m} \sigma_{k}^{2}}, \\
\mathcal{N}_{a_{n}, \sigma_{n}^{2}} * \cdots * \mathcal{N}_{a_{n+m}, \sigma_{n+m}^{2}} * \delta_{a} & =\mathcal{N}_{a+\sum_{k=n}^{n+m} a_{k}, \sum_{k=n}^{n+m} \sigma_{k}^{2}} \quad \text { and } \\
H^{2}\left(\mathcal{N}_{a+\sum_{k=n}^{n+m} a_{k}, \sum_{k=n}^{n+m} \sigma_{k}^{2}}, \mathcal{N}_{\sum_{k=n}^{n+m} a_{k}, \sum_{5=n}^{n+m} \sigma_{k}^{2}}\right) & =1-e^{-\frac{1}{8} \cdot \frac{a^{2}}{\sum_{k=n}^{n+m} \sigma_{k}^{2}}} \rightarrow 0
\end{aligned}
$$


as $m \rightarrow \infty^{2}$. Hence $\left(\xi_{n}\right)_{n=1}^{\infty}$ is ATI in view of $(1-1)$.

1.4. Ergodic cocycles of ergodic transformation groups. Given a standard Borel $\sigma$-finite measure space $(Y, \mathfrak{C}, \nu)$, we denote by $\operatorname{Aut}(Y, \nu)$ the group of all $\nu$-nonsingular invertible Borel transfomations on $Y$. Let $\operatorname{Aut}_{0}(Y, \nu)$ denote the subgroup of $\nu$-preserving transformations from $\operatorname{Aut}(Y, \nu)$. Let $\Gamma$ be an ergodic countable subgroup in $\operatorname{Aut}(Y, \nu)$. The full group $[\Gamma]$ of $\Gamma$ is defined by:

$$
[\Gamma]:=\{\theta \in \operatorname{Aut}(Y, \nu) \mid \theta y \in\{\gamma y \mid \gamma \in \Gamma\} \text { at a.e. } y \in Y\} .
$$

A measurable map $\alpha: \Gamma \times Y \rightarrow G$ is called a cocycle of $\Gamma$ if

$$
\alpha\left(\gamma_{1} \gamma_{2}, y\right)=\alpha\left(\gamma_{1}, \gamma_{2} y\right)+\alpha\left(\gamma_{2}, y\right) \quad \text { at a.e. } y \in Y
$$

for all $\gamma_{1}, \gamma_{2} \in \Gamma$. From now on we assume that $\Gamma$ is free, i.e. if $\gamma \in \Gamma \backslash\{I\}$ then $\gamma y \neq y$ for a.e. $y$. Then $\alpha$ can be "extended" to $[\Gamma]$ if we set

$$
\alpha(\theta, y):=\alpha(\gamma, y) \quad \text { where } \gamma \text { is defined uniquely by } \theta y=\gamma y .
$$

It is straightforward to verify that (1-3) holds if we replace $\gamma_{1}$ and $\gamma_{2}$ with arbitrary elements from $[\Gamma]$. A cocycle $\alpha$ is a coboundary if there is a measurable map $a: Y \rightarrow G$ such that

$$
\alpha(\gamma, y)=a(\gamma y)-a(y) \quad \text { at a.e. } y \in Y
$$

for all $\gamma \in \Gamma$. Given a pair $(\Gamma, \alpha)$, we can construct a transformation group $\Gamma_{\alpha}:=$ $\left\{\gamma_{\alpha} \mid \gamma \in \Gamma\right\} \subset \operatorname{Aut}\left(Y \times G, \nu \times \lambda_{G}\right)$, where

$$
\gamma_{\alpha}(y, g):=(\gamma y, \alpha(\gamma, y)+g) \quad \text { for all } y \in Y, g \in G .
$$

The group $\Gamma_{\alpha}$ is called the $\alpha$-skew product extension of $\Gamma$. If $\Gamma$ preserves $\nu$ then $\Gamma_{\alpha}$ preserves the product measure $\nu \otimes \lambda_{G}$. If $\Gamma_{\alpha}$ is ergodic then $\alpha$ is called ergodic. A coboundary is never ergodic (unless $G$ is a singleton). It is easy to verify that if $\Gamma=\left\{R^{n} \mid n \in \mathbb{Z}\right\}$ for a transformation $R \in \operatorname{Aut}(Y, \nu)$ then each measurable function $f: Y \rightarrow G$ defines uniquely a cocycle $\alpha_{f}$ of $\Gamma$ via the condition

$$
\alpha_{f}(R, y):=f(y) \quad \text { for each } y \in Y .
$$

For brevity we will write $R_{f}$ for the $\alpha_{f}$-skew product extension $R_{\alpha_{f}}$ of $R$.

We now recall an important concept of essential value for a cocycle.

Definition 1.4. Suppose that $\Gamma$ preserves $\nu$. An element $g \in G$ is called an essential value of $\alpha$ if for each subset $A \subset Y$ of positive measure and a neighborhood $U$ of $g$, there are a Borel subset $B \subset A$ and an element $\gamma \in \Gamma$ such that $\nu(B)>0$, $\gamma B \subset A$ and $\alpha(\gamma, y) \in U$ for all $y \in B$.

It appears that the set $r(\alpha)$ of all essential values of a cocycle is a closed subgroup of $G$. Our interest to the essential values of $\alpha$ is explained by the fact that $\alpha$ is ergodic if and only if $r(\alpha)=G$ [Sc]. It is often easier to check the aforementioned condition on essential values not for each subset $A \in \mathfrak{C}$ of positive measure but only for a dense subfamily of subsets in $\mathfrak{C}$. However in this case we have to strengthen this condition. More precisely, we will use the following lemma. (It follows, for example, from [Da, Lemma 2.4].)

${ }^{2}$ We use the fact that $H^{2}\left(\mathcal{N}_{a, \sigma^{2}}, \mathcal{N}_{b, \tau^{2}}\right)=1-\sqrt{\frac{2 \sigma \tau}{\sigma^{2}+\tau^{2}}} e^{-\frac{1}{4} \frac{(a-b)^{2}}{\sigma^{2}+\tau^{2}}}$ for all $a, b, \sigma, \tau \in \mathbb{R}$. 
Lemma C. Let $(Y, \mathfrak{C}, \nu)$ be a standard probability space, $\mathfrak{A}$ a dense subset in $\mathfrak{C}, \Gamma$ an ergodic countable subgroup of $\operatorname{Aut}_{0}(Y, \nu)$ and $\alpha: \Gamma \times Y \rightarrow G$ a Borel cocycle of $\Gamma$. If for some $a \in G$ and each subset $B \in \mathfrak{A}$ and each neighborhood $U$ of 0 in $G$, there are a measurable subset $D \subset B$ and an element $\theta \in[\Gamma]$ such that $\theta D \subset B$, $\nu(D)>0.5 \nu(B)$ and $\alpha(\theta, x) \in a+U$ for all $x \in D$ then $a$ is an essential value of $\alpha$.

1.5. Sharp weak mixing of skew products for cocycles of product type. In this subsection we prove the following theorem.

Theorem 1.5. Let $T_{n}$ be a mildly mixing transformation of a standard probability space $\left(X_{n}, \mathfrak{B}_{n}, \nu_{n}\right)$ for each $n \in \mathbb{N}$. Let

$$
(X, \mathfrak{B}, \nu, T):=\bigotimes_{n \in \mathbb{Z}}\left(X_{n}, \mathfrak{B}_{n}, \nu_{n}, T\right)
$$

Suppose that for a measurable function $f: X \rightarrow G$, there are functions $f_{n}: X_{n} \rightarrow G$ such that $f(x)=\sum_{n \in \mathbb{N}}\left(f_{n}\left(T_{n} x_{n}\right)-f_{n}\left(x_{n}\right)\right)$ at $\nu$-a.e. $x=\left(x_{n}\right)_{n=1}^{\infty} \in X$ and the sequence of measures $\left(\nu_{n} \circ f_{n}^{-1}\right)_{n \in \mathbb{N}}$ is ATI. If the skew product extension $T_{f}$ : $X \times G \rightarrow X \times G$ of $T$ is conservative then $T_{f}$ is sharply weak mixing.

Proof. Let $C$ be an ergodic conservative transformation of a standard probability space $(Z, \mathfrak{Z}, \kappa)$. Suppose that $\left(\mu \otimes \lambda_{G} \otimes \kappa\right)\left(\mathcal{C}\left(T_{f} \times C\right)\right)>0$. Since $T_{f} \times C$ commutes with $I \times C$,

$$
(I \times C) \mathcal{C}\left(T_{f} \times C\right)=\mathcal{C}\left(T_{f} \times C\right) .
$$

Since $C$ is ergodic, $\mathcal{C}\left(T_{f} \times C\right)=\mathcal{C}\left(T_{f}\right) \times Z=X \times G \times Z$, where the latter equality holds because $T_{f}$ is conservative. Thus, the direct product $T_{f} \times C$ is conservative. It remains to show that $T_{f} \times C$ is ergodic.

Let a function $F \in L^{\infty}\left(X \times G \times Z, \mu \otimes \lambda_{G} \otimes \kappa\right)$ be invariant under $T_{f} \times C$. We first show that $F$ is also invariant under a huge group of transformations. Fix $n>0$. For each $x \in X$, we write $x_{1}^{n}:=\left(x_{1}, \ldots, x_{n}\right) \in X_{1} \times \cdots \times X_{n}$ and $x_{n+1}^{\infty}:=\left(x_{n+1}, x_{n+2}, \ldots\right) \in X_{n+1} \times X_{n+2} \times \cdots$. Then $x=\left(x_{1}^{n}, x_{n+1}^{\infty}\right)$. We define a measure preserving automorphism $E_{n}$ of $\left(X \times G \times Z, \mu \otimes \lambda_{G} \otimes \kappa\right)$ and a nonsingular automorphism $V_{n}$ of $\left(\bigotimes_{k=n+1}^{\infty}\left(X_{k}, \nu_{k}\right)\right) \otimes\left(G, \lambda_{G}\right) \otimes(Z, \kappa)$ respectively by setting

$$
\begin{aligned}
E_{n}(x, g, z) & :=\left(x, g+\sum_{k=1}^{n} f_{k}\left(x_{k}\right), z\right) \text { and } \\
V_{n}\left(\left(x_{k}\right)_{k=n+1}^{\infty}, g, z\right) & :=\left(\left(T_{k} x_{k}\right)_{k=n+1}^{\infty}, g+\sum_{k>n}\left(f_{k}\left(T_{k} x_{k}\right)-f_{k}\left(x_{k}\right)\right), C z\right) .
\end{aligned}
$$

A straightforward verification shows that

$$
E_{n}\left(T_{f} \times C\right) E_{n}^{-1}=\left(T_{1} \times \cdots \times T_{n}\right) \times V_{n} .
$$

Since $V_{n}$ is a factor of the transformation $E_{n}\left(T_{f} \times C\right) E_{n}^{-1}$ and the latter transformation is conservative, it follows that $V_{n}$ is conservative. On the other hand, the function $F \circ E_{n}$ is invariant under $E_{n}^{-1}\left(T_{f} \times C\right) E_{n}$. Utilizing these two facts we deduce from Theorem $\mathrm{B}$ that $F \circ E_{n}$ does not depend on the coordinates $x_{1}, \ldots, x_{n}$. Hence, for each transformation $S \in \operatorname{Aut}_{0}\left(X_{1} \times \cdots \times X_{n}, \bigotimes_{k=1}^{n} \nu_{k}\right)$, we have that 
$F \circ E_{n} \circ(S \times I)=F \circ E_{n}$. Therefore $F$ is invariant under the transformation $E_{n}(S \times I) E_{n}^{-1} \in \operatorname{Aut}_{0}\left(X \times G \times Z, \mu \times \lambda_{G} \times \kappa\right)$ and

$$
E_{n}(S \times I) E_{n}^{-1}(x, g, z)=\left(S x_{1}^{n}, x_{n+1}^{\infty}, g-A_{n}\left(x_{1}^{n}\right)+A_{n}\left(S x_{1}^{n}\right), z\right),
$$

where $A_{n}$ stands for the mapping $X_{1} \times \cdots \times X_{n} \ni\left(x_{1}, \ldots, x_{n}\right) \mapsto \sum_{k=1}^{n} f_{k}\left(x_{k}\right)$. Thus, we have shown that $F$ is invariant under each transformation from the set

$$
\mathcal{G}:=\bigcup_{n>0} E_{n}\left(\operatorname{Aut}_{0}\left(X_{1} \times \cdots \times X_{n}, \bigotimes_{k=1}^{n} \nu_{k}\right) \times\{I\}\right) E_{n}^{-1}
$$

We now consider a new dynamical system. The space of this system is the product $(X, \mathfrak{B}, \mu)$. Denote by $\Gamma$ the group of transformations of this space generated by mutually commuting measure preserving transformations $\widehat{T}_{1}, \widehat{T}_{2}, \ldots$, where

$$
\widehat{T}_{n} x=\left(x_{1}^{n-1}, T_{n} x_{n}, x_{n+1}^{\infty}\right), \quad n \in \mathbb{N} .
$$

Then $\Gamma$ is countable, Abelian ${ }^{3}$ and ergodic. For each $n>0$, we consider a coboundary

$$
\alpha_{n}: X \ni x \mapsto \alpha_{n}(x):=f_{n}\left(T_{n} x_{n}\right)-f_{n}\left(x_{n}\right) \in G
$$

of $\widehat{T}_{n}$. It is straightforward to verify ${ }^{4}$ that the $\alpha_{n}$-skew product extensions $\left(\widehat{T}_{n}\right)_{\alpha_{n}}$ of $\widehat{T}_{n}, n \in \mathbb{N}$, commute mutually. It follows that a cocycle $\alpha: \Gamma \times X \rightarrow G$ of $\Gamma$ with values in $G$ is well defined by the following formulae:

$$
\alpha\left(\widehat{T}_{n}, x\right):=\alpha_{n}(x), \quad n \in \mathbb{N} .
$$

Since $\alpha_{n}(x)=A_{n}\left(\left(I \times T_{n}\right) x_{1}^{n}\right)-A_{n}\left(x_{1}^{n}\right)$, it follows from (1-4) that

$$
E_{n}\left(I \times T_{n} \times I\right) E_{n}^{-1}=\left(\widehat{T}_{n}\right)_{\alpha_{n}} \times I_{Z} .
$$

Hence $\left(\widehat{T}_{n}\right)_{\alpha_{n}} \times I_{Z} \in \mathcal{G}$. Although, each $\alpha_{n}$ is a coboundary for the $\mathbb{Z}$-action given by $T_{n}$, the cocycle $\alpha$ is not a coboundary for $\Gamma$. In fact, we will now show the following.

Claim I. The cocycle $\alpha$ of $\Gamma$ is ergodic.

For that we will show that each element $a \in G$ is an essential value of $\alpha$. Given $n>0$ and a subset $B \subset X_{1} \times \cdots \times X_{n}$, denote by $[B]_{1}^{n} \subset X$ the corresponding cylinder with the "head" $B$, i.e. $[B]_{1}^{n}:=\left\{x \in X \mid x_{1}^{n} \in B\right\}$. Let $U$ be a symmetric neighborhood of 0 in $G$. Choose a countable partition $\mathcal{P}$ of $G$ into Borel subsets $\Delta$ such that $g-h \in U$ for all $g, h \in \Delta$ and each $\Delta \in \mathcal{P}$. Let $\psi_{k}:=\nu_{k} \circ f_{k}^{-1}$ for each $k>0$. Using the ATI-assumption, we can find $m>n$ such that

$$
\left\|\psi_{n+1} * \cdots * \psi_{m} * \delta_{a}-\psi_{n+1} * \cdots * \psi_{m}\right\|_{1}<\epsilon .
$$

\footnotetext{
${ }^{3}$ It is isomorphic to $\bigoplus_{n=1}^{\infty} \mathbb{Z}$.

${ }^{4}$ This follows from the fact that each function $\alpha_{n}$ depends only on a single coordinate $x_{n}$, $n=1,2, \ldots$.
} 
For each $\Delta \in \mathcal{P}$, we let

$$
\begin{aligned}
& A_{\Delta}:=\left\{y=\left(y_{k}\right)_{k=n+1}^{m} \in X_{n+1} \times \cdots \times X_{m} \mid \sum_{k=n+1}^{m} f_{k}\left(y_{k}\right) \in \Delta\right\} \text { and } \\
& B_{\Delta}:=\left\{y=\left(y_{k}\right)_{k=n+1}^{m} \in X_{n+1} \times \cdots \times X_{m} \mid a+\sum_{k=n+1}^{m} f_{k}\left(y_{k}\right) \in \Delta\right\} .
\end{aligned}
$$

Then $\left\{A_{\Delta}\right\}_{\Delta \in \mathcal{P}}$ and $\left\{B_{\Delta}\right\}_{\Delta \in \mathcal{P}}$ are two measurable partitions of $X_{n+1} \times \cdots \times X_{m}$. It follows from (1-5) that

$$
\begin{aligned}
\sum_{\Delta \in \mathcal{P}}\left|\nu_{n+1}^{m}\left(A_{\Delta}\right)-\nu_{n+1}^{m}\left(B_{\Delta}\right)\right| & =\sum_{\Delta \in \mathcal{P}}\left|\psi_{n+1} * \cdots * \psi_{m} * \delta_{a}(\Delta)-\psi_{n+1} * \cdots * \psi_{m}(\Delta)\right| \\
& \leq\left\|\psi_{n+1} * \cdots \psi_{m} * \delta_{a}-\psi_{n+1} * \cdots * \psi_{m}\right\|_{1} \\
& <\epsilon,
\end{aligned}
$$

where $\nu_{n+1}^{m}$ denotes the direct product $\bigotimes_{k=n+1}^{m} \nu_{k}$. We can find subsets $A_{\Delta}^{\prime} \subset A_{\Delta}$ and $B_{\Delta}^{\prime} \subset B_{\Delta}$ such that

$$
\nu_{n+1}^{m}\left(A_{\Delta}^{\prime}\right)=\nu_{n+1}^{m}\left(B_{\Delta}^{\prime}\right)=\min \left(\nu_{n+1}^{m}\left(A_{\Delta}\right), \nu_{n+1}^{m}\left(B_{\Delta}\right)\right) .
$$

Note that the group $\Gamma_{n+1, m}$ generated by $m-n$ mutually commuting transformations $T_{n+1} \times I \times \cdots \times I, I \times T_{n+2} \times I \times \cdots \times I, \ldots, I \times \cdots \times I \times T_{m} \in$ $\operatorname{Aut}_{0}\left(X_{n+1} \times \cdots \times X_{m}, \nu_{n+1}^{m}\right)$ is ergodic. Hence, in view of (1-7), Hopf's lemma $[\mathrm{HaOs}]$ yields that there is a transformation $S_{0} \in\left[\Gamma_{n+1, m}\right]$ such that $S_{0} A_{\Delta}^{\prime}=B_{\Delta}^{\prime}$ for each $\Delta \in \mathcal{P}$. We note that

$$
\sum_{\Delta \in \mathcal{P}} \nu_{n+1}^{m}\left(A_{\Delta} \backslash A_{\Delta}^{\prime}\right) \leq \sum_{\Delta \in \mathcal{P}}\left|\nu_{n+1}^{m}\left(A_{\Delta}\right)-\nu_{n+1}^{m}\left(B_{\Delta}\right)\right|<\epsilon .
$$

It follows that $\nu_{n+1}^{m}\left(\bigsqcup_{\Delta \in \mathcal{P}} A_{\Delta}^{\prime}\right)>1-\epsilon$. On the other hand, in view of (1-6), for each $y \in A^{+}:=\bigsqcup_{\Delta \in \mathcal{P}} A_{\Delta}^{\prime}$,

$$
\left(\sum_{k=n+1}^{m} f_{k}\right)(y)-\left(\sum_{k=n+1}^{m} f_{k}\right)\left(S_{0} y\right) \in a+U
$$

We now "extend" $S_{0}$ to a transformation $S \in \operatorname{Aut}_{0}(X, \mu)$ by setting

$$
S x:=\left(x_{1}^{n}, S_{0} x_{n+1}^{m}, x_{m+1}^{\infty}\right) \in X \quad \text { for all } x \in X .
$$

Then $S \in[\Gamma]$ and

$$
\alpha(S, x) \in a+U \quad \text { whenever } x_{n+1}^{m} \in A^{+} .
$$

Then we have that $\left[B \times A^{+}\right]_{1}^{m} \subset[B]_{1}^{n}, S\left[B \times A^{+}\right]_{1}^{m} \subset[B]_{1}^{n}, \mu\left(\left[B \times A^{+}\right]_{1}^{m}\right)>\frac{1}{2} \mu\left([B]_{1}^{n}\right)$ and (1-8) holds for all $x \in\left[B \times A^{+}\right]_{1}^{m}$. Since the set of all cylinders is dense in $\mathfrak{B}$, it follows from Lemma $\mathrm{C}$ that $a$ is an essential value of $\alpha$. Thus, Claim I is proved.

To complete the proof of the theorem, we have already noticed that $\left(\widehat{T}_{n}\right)_{\alpha_{n}} \times I_{Z} \in$ $\mathcal{G}$ for each $n \in \mathbb{N}$. Hence $F\left(\gamma_{\alpha}(x, g), z\right)=F(x, g, z)$ at a.e. $(x, g, z) \in X \times G \times Z$ for each $\gamma \in \Gamma$. Claim I yields that there is a function $M: Z \rightarrow \mathbb{R}$ such that $F(x, g, z)=M(z)$ at a.e. $(x, g, z) \in X \times G \times Z$. Since $F$ is invariant under $T_{f} \times C$, we obtain that $M$ is invariant under $C$. Since $C$ is ergodic, $M$ is constant a.e. and hence $F$ is constant a.e., i.e. $T_{f} \times C$ is ergodic.

We call the cocycle $f$ in the statement of Theorem 1.5 a cocycle of product type. 
1.6. Application to Gaussian cocycles. Let $(X, \mathfrak{B}, \mu, T)$ be an ergodic Gaussian dynamical system. It is completely determined by a restriction of the corresponding Koopman unitary operator $U_{T}$ to a closed (real) Gaussian subspace $H \subset L_{0}^{2}(X, \mu)$, called the first chaos. (See, e.g. [LePaTh] for the definitions.) Let $\kappa$ denote the maximal spectral type of $U_{T} \uparrow H$. It is known that $T$ is ergodic if and only if $T$ is weakly mixing if and only if $\kappa$ is nonatomic. Take $f \in H$. Then the measurable map $f: X \rightarrow \mathbb{R}$ considered as a cocycle of $T$ is called a Gaussian cocycle. It was shown in [LeLeSk] that if $f$ is a $T$-coboundary, i.e. $f=h \circ T-h$ for a measurable function $h: X \rightarrow \mathbb{R}$, then $h \in H$. We now recall a conjecture from [LeLeSk].

Conjecture I. If a Gaussian cocycle $f$ is not a coboundary then $f$ is ergodic.

We now prove this conjecture (in fact, we prove a stronger result) under an additional assumption that $T$ is mildly mixing.

Theorem 1.6. If $T$ is a mildly mixing Gaussian transformation and $f$ is a Gaussian cocycle of $T$ which is not a coboundary then $T_{f}$ is sharply weak mixing.

Proof. Since $f \in H$, it follows that $\int_{X} f d \mu=0$. Hence, by Atkinson's theorem [At], $T_{f}$ is conservative. Consider now the spectral decomposition for the pair $\left(H, U_{T}\right)$ :

$$
H=\int_{\mathbb{T}}^{\oplus} \mathcal{H}_{z} d \kappa(z) \quad \text { and } \quad U_{T}=\int_{\mathbb{T}}^{\oplus} z I_{z} d \kappa(z),
$$

where $\mathbb{T} \ni z \mapsto \mathcal{H}_{z}$ is the corresponding measurable field of Hilbert spaces and $I_{z}$ is the identity operator in $\mathcal{H}_{z}$. In other words, we can consider an element $h$ of $H$ as a measurable map $\mathbb{T} \ni z \mapsto h(z) \in \mathcal{H}_{z}$ such that $\|h\|^{2}=\int_{\mathbb{T}}\|h(z)\|^{2} d \kappa(z)<\infty$. We now let $\Delta_{n}:=\left\{z \in \mathbb{T}\left|\frac{1}{n+1}<\right| z-1 \mid \leq \frac{1}{n}\right\}$. Then we obtain a countable partition $\bigsqcup_{n=1}^{\infty} \Delta_{n}$ of $\mathbb{T} \backslash\{1\}$. Since $\kappa(\{1\})=0$, this countable partition generates a decomposition of $H$ into a direct sum $\bigoplus_{n \in \mathbb{N}} H_{n}$ of closed $U_{T}$-invariant subspaces $H_{n}$ consisting of the measurable maps $h: \mathbb{T} \ni z \mapsto h(z) \in \mathcal{H}_{z}$ such that $h(z)=0$ whenever $z \notin \Delta_{n}$. This decomposition induces a decomposition of $(X, \mu, T)$ into the infinite direct product $(X, \mu, T)=\bigotimes_{n=1}^{\infty}\left(X_{n}, \mu_{n}, T_{n}\right)$, where $\left(X_{n}, \mu_{n}, T_{n}\right)$ is the Gaussian dynamical system associated with the pair $\left(H_{n}, U_{T}\left\lceil H_{n}\right)\right.$ for each $n \in \mathbb{N}$. Now we can expand $f$ into an orthogonal sum $f=\bigoplus_{n=1}^{\infty} f_{n}$ with $f_{n} \in H_{n}$ for each $n \in \mathbb{N}$. Of course, for each $n>0$, there is $a_{n} \in H_{n}$ such that $f_{n}=U_{T} a_{n}-a_{n}$. Indeed, it follows from this equation that $f_{n}(z)=z a_{n}(z)-a_{n}(z)$ and hence $a_{n}(z)=$ $(z-1)^{-1} f_{n}(z)$ for a.e. $z \in \Delta_{n}$. Since $|z-1|^{-1}<n+1$ for all $z \in \Delta_{n}$, we obtain that $a_{n} \in H_{n}$. This yields an expansion

$$
f=\bigoplus_{n=1}^{\infty}\left(U_{T} a_{n}-a_{n}\right)=\bigoplus_{n=1}^{\infty}\left(a_{n} \circ T_{n}^{-1}-a_{n}\right)
$$

of $f$ into an infinite sum of $T_{n}$-coboundaries. Of course, $\sum_{n \in \mathbb{N}}\left\|a_{n}\right\|^{2}=+\infty$. Otherwise the series $\sum_{n \in \mathbb{N}} a_{n}$ converges in $H$ and hence $f$ would be a coboundary which contradicts the assumption of the theorem. We have that $\mu_{n} \circ a_{n}^{-1}=\mathcal{N}_{0,\left\|a_{n}\right\|^{2}}$ for each $n \in \mathbb{N}$. Passing, if necessary, to a subsequence we may assume without loss of generality that the convergence in (1-9) is almost everywhere. Example 1.3 yields that the sequence $\left(\mu_{n} \circ a_{n}^{-1}\right)_{n=1}^{\infty}$ is ATI. It now follows from Theorem 1.5 that $T_{f}$ is sharply weak mixing. 
Consider now the general case. Then there is a maximal (with respect to $\kappa$ ) subset $A$ of $\mathbb{T}$ such that $U_{T}$ restricted to the closed subspace $\int_{A}^{\oplus} \mathcal{H}_{z} d \kappa(z)$ of $H$ is mildly mixing. We note that $A$ is symmetric. Then $\kappa$ decomposes into a sum of two orthogonal measures: $\kappa_{m m}:=\kappa \uparrow A$ (the mildly mixing part of $\kappa$ ) and $\kappa_{r}:=\kappa \uparrow(\mathbb{T} \backslash A$ ) (the rigid part of $\kappa)$. This decomposition defines a decomposition of $(X, \mu, T)$ into a direct product $\left(X_{1}, \mu_{m m}, M\right) \times\left(X_{2}, \mu_{r}, R\right)$, where $\left(X_{1}, \mu_{m m}, M\right)$ is the Gaussian dynamical system corresponding to the pair $\left(\int_{A}^{\oplus} \mathcal{H}_{z} d \kappa_{m m}(z), U_{T}\right)$ and $\left(X_{2}, \mu_{r}, R\right)$ is the Gaussian dynamical system corresponding to the pair $\left(\int_{\mathbb{T} \backslash A}^{\oplus} \mathcal{H}_{z} d \kappa_{r}(z), U_{T}\right)$. Also, we obtain a decomposition of $f$ into a sum $f_{m m}+f_{r}$, where $f_{m m}:=f 1_{A}$ and $f_{r}=f 1_{\mathbb{T} \backslash A}$. There are two possible cases: either $f_{m m}$ is a coboundary or $f_{m m}$ is not a coboundary. In the first case $T_{f}$ is isomorphic to $Q \times R_{f_{r}}$. Moreover, $f_{r}$ is not a coboundary because otherwise $f$ would be a coboundary. Since $Q$ is mildly mixing and $T_{f}$ is conservative, $T_{f}$ is ergodic if and only if $R_{f_{r}}$ is ergodic. In the second case, $T_{f}$ is isomorphic to $Q_{f_{m m}} \times R_{f_{r}}$ and $Q_{f_{m m}}$ is sharply weak mixing by Theorem 1.5. Since $T_{f}$ is conservative, it follows that $T_{f}$ is ergodic if and only if $R_{f_{r}}$ is ergodic. Thus, we have reduced the conjecture from [LeLeSk] to the following one.

Conjecture II. If a Gaussian cocycle $f$ is not a coboundary and $\kappa$ has only rigid part then $f$ is ergodic. ${ }^{5}$

\section{KRIEGER'S TYPE OF INFINITE DIRECT PRODUCTS OF DYNAMICAL SYSTEMS OF FINITE TYPE}

2.1. IDPFT systems. Let $T_{n}$ be a nonsingular invertible transformation of a standard probability space $\left(X_{n}, \mathfrak{B}_{n}, \mu_{n}\right)$ for each $n \in \mathbb{N}$. Denote by $T$ the infinite direct product of $T_{n}, n \in \mathbb{N}$, acting on the infinite product space $(X, \mathfrak{B}, \mu):=$ $\bigotimes_{n \in \mathbb{Z}}\left(X_{n}, \mathfrak{B}_{n}, \mu_{n}\right)$. By Theorem A, $T$ is $\mu$-nonsingular if and only if

$$
\prod_{n=1}^{\infty}\left(1-H^{2}\left(\mu_{n} \circ T_{n}^{-1}, \mu_{n}\right)\right)>0 \text { or } \sum_{n=1}^{\infty} H^{2}\left(\mu_{n} \circ T_{n}^{-1}, \mu_{n}\right)<\infty .
$$

If (2-1) does not hold then $\mu \circ T^{-1} \perp \mu$. If $T$ is $\mu$-nonsingular then

$$
\frac{d \mu \circ T^{-1}}{d \mu}(x)=\prod_{n=1}^{\infty} \frac{d \mu_{n} \circ T_{n}^{-1}}{d \mu_{n}}\left(x_{n}\right) \quad \text { at a.e. } x \in X .
$$

Suppose now that $T_{n}$ is of finite type, i.e that there exists a $\mu_{n}$-equivalent probability measure $\nu_{n}$ which is invariant under $T_{n}$ for each $n \in \mathbb{N}$. We then put $\phi_{n}:=\frac{d \mu_{n}}{d \nu_{n}}$. Since $1-H^{2}\left(\mu_{n} \circ T_{n}^{-1}, \mu_{n}\right)=\int_{X_{n}} \sqrt{\frac{\phi_{n} \circ T_{n}^{-1}}{\phi_{n}}} \phi_{n} d \nu_{n}$, the formula (2-1) and Theorem A yield the following.

Corollary 2.1. $T$ is $\mu$-nonsingular if and only if

$$
\prod_{n=1}^{\infty} \int_{X_{n}} \sqrt{\phi_{n} \cdot \phi_{n} \circ T_{n}^{-1}} d \nu_{n}>0 .
$$

\footnotetext{
${ }^{5}$ In [LeLeSk], there were constructed some concrete rigid Gaussian transformations admitting ergodic Gaussian cocycles. In [MaRa] this result was extended to arbitrary rigid Gaussian transformations which have at least one Gaussian non-coboundary. However, it is unknown whether the ergodicity holds for each Gaussian non-coboundary in those examples.
} 
$\mu \perp \nu$ if and only if

$$
\prod_{n=1}^{\infty} \int_{X_{n}} \sqrt{\phi}_{n} d \nu_{n}=0
$$

Definition 2.2. If $T$ is $\mu$-nonsingular and $T_{n}$ is of finite type for all $n>0$ then we say that the dynamical system $(X, \mathfrak{B}, \mu, T)$ is IDPFT (i.e. infinite direct product of finite types).

Our purpose in this section is to investigate dynamical properties of IDPFTsystems. The first result is about ergodicity of conservative IDPFT systems under the mild mixing assumption on the factors.

Proposition 2.3. Let $\left(X_{n}, \nu_{n}, T_{n}\right)$ be mildly mixing for each $n>0$ and (2-2) and (2-3) hold. Suppose that $T$ is $\mu$-conservative. Then $T$ is $\mu$-sharply weak mixing and $\mu \perp \nu$.

Proof. Let $S$ be an ergodic conservative transformation of a standard probability space $(Y, \mathfrak{C}, \nu)$. As in the proof of Theorem 1.5 one can show that $T \times S$ is either totally dissipative or conservative. Suppose that $T \times S$ is conservative. We have to prove that it is ergodic. Let a subset $A \in \mathfrak{B} \otimes \mathfrak{C}$ be invariant under $T \times S$. It follows from Theorem B that for each $n>0, A$ belongs to the $\sigma$-algebra $\left\{\emptyset, X_{1} \times \cdots \times\right.$ $\left.X_{n}\right\} \otimes \mathfrak{B}_{n+1} \otimes \mathfrak{B}_{n+2} \otimes \cdots \otimes \mathfrak{C}^{6}$. By the Kolmogorov 0-1 law, the intersection of these $\sigma$-algebras is $\mathfrak{N} \otimes \mathfrak{C}$, where $\mathfrak{N}$ is the trivial $\sigma$-algebra on $X$. Thus $A=X \times D$ for some subset $D \in \mathfrak{C}$. Since $A$ in invariant under $T \times S$, it follows that $D$ is invariant under $S$. Since $S$ is ergodic, we obtain that either $\mu \otimes \nu(A)=0$ or $\mu \otimes \nu(A)=1$.

Remark 2.4. In Section 3 below we will give examples of $(X, \nu, T)$ and $\mu$ such that $(X, \mu, T)$ is of type $I I I_{1}$. In particular, there is no $\mu$-equivalent invariant probability measure. On the other hand, we do not know examples in which $(X, \mu, T)$ is of type $I I_{1}$, i.e. $T$ is mildly mixing with respect to a $\mu$-equivalent invariant probability measure.

2.2. Radon-Nikodym cocycle and type $I I I_{1}$. Let $\Gamma$ be an ergodic countable subgroup of $\operatorname{Aut}(Y, \nu)$. Denote by $\rho_{\nu}: \Gamma \times Y \rightarrow \mathbb{R}$ the logarithm of the RadonNikodym cocycle of $\Gamma$, i.e.

$$
\rho_{\nu}(\gamma, y):=\log \frac{d \nu \circ \gamma}{d \nu}(y)
$$

The $\rho_{\nu}$-skew product extension $\Gamma_{\rho_{\nu}}$ of $\Gamma$ is called the Maharam extension of $R$. We note that $\Gamma_{\rho_{\nu}}$ preserves an equivalent $\sigma$-finite measure $\nu \otimes \kappa$, where $\kappa$ is a Lebesgue absolutely continuous $\sigma$-finite measure on $\mathbb{R}$ such that $d \kappa(t)=e^{-t} d t$ for all $t \in \mathbb{R}$. Similar to the finite measure preserving case, $\rho_{\nu}$ "extends" to the full group $[\Gamma]$ in such a way that the cocycle identity holds. Moreover, we do not need the freeness condition for $\Gamma$ to define this extension.

We note that $\rho_{\nu}$ is a coboundary if and only if there is a $\Gamma$-invariant $\nu$-equivalent $\sigma$-finite measure on $(Y, \mathfrak{C})$.

\footnotetext{
${ }^{6}$ When applying Theorem B, we consider the measure $\left(\otimes_{k=1}^{n} \nu_{k}\right) \otimes\left(\otimes_{k>n} \mu_{k}\right) \otimes \nu$ on $X \times Y$. This measure is equivalent to $\mu \otimes \nu$.
} 
By the Maharam theorem (see [Sc]), $\Gamma_{\rho_{\nu}}$ is conservative if and only if $\Gamma$ is conservative. However if $\Gamma$ is ergodic then $\Gamma_{\rho_{\nu}}$ is not necessarily ergodic. If the Maharam extension of $\Gamma$ is ergodic then $\Gamma$ is called of Krieger's type $I I I_{1}$. If for each homomorphism $\vartheta: \Gamma \rightarrow \operatorname{Aut}_{0}(Y, \nu)$ such that the image $\{\vartheta(\gamma) \mid \gamma \in \Gamma\}$ is ergodic, the direct product $\{\gamma \times \vartheta(\gamma) \mid \gamma \in \Gamma\}$ is ergodic and of type $I I I_{1}$ then $\Gamma$ is said to be of stable Krieger's type $I I I_{1}$.

It is possible to define essential values of $\rho_{\nu}$ in the same way as in the finite measure preserving case.

Definition 2.5. An element $g \in \mathbb{R}$ is called an essential value of $\rho_{\nu}$ if for each subset $A \subset Y$ of positive measure and a neighborhood $U$ of $g$, there are a Borel subset $B \subset A$ and an element $\gamma \in \Gamma$ such that $\nu(B)>0, \gamma B \subset A$ and $\rho_{\nu}(\gamma, y) \in U$ for all $y \in B$.

We refer to $[\mathrm{Sc}]$ and $[\mathrm{HaOs}]$ for the proof of the following results:

- The set $r\left(\rho_{\nu}\right)$ is a closed subgroup in $\mathbb{R}$.

- $\Gamma$ is of type $I I I_{1}$ if and only if $r\left(\rho_{\nu}\right)=\mathbb{R}$.

If there is $\lambda \in(0,1)$ such that $r\left(\rho_{\nu}\right)=\{n \log \lambda \mid n \in \mathbb{Z}\}$ then $\Gamma$ is said to be of Krieger's type $I I I_{\lambda}$.

We will need the following analog of Lemma C. It follows from a more general [Da, Lemma 2.4].

Lemma D. An element $a \in \mathbb{R}$ is an essential value of $\rho_{\nu}$ if there exists $\delta>0$ such that for each $\epsilon>0$ and each subset $B$ from a dense collection $\mathfrak{C}_{0}$ of subsets in $\mathfrak{C}$, there is a subset $B_{0} \subset B$ and a transformation $\theta \in[\Gamma]$ such that $\nu\left(B_{0}\right)>\delta \nu(B)$, $\theta B_{0} \subset B$ and either $\left|\rho_{\nu}(\theta, y)-a\right| \leq \epsilon$ for all $y \in B_{0}$ or $\left|\rho_{\nu}(\theta, y)+a\right| \leq \epsilon$ for all $y \in B_{0}$.

2.3. On conservativeness of IDPFT systems. In this subsection we first establish a general result on conservativeness of infinite direct product systems.

Proposition 2.6. Let $\left(X_{n}, \mathfrak{B}_{n}, \mu_{n}, T_{n}\right)$ be an ergodic nonsingular dynamical system on a standard probability space for each $n \in \mathbb{N}$ and let $(2-1)$ hold. Let $(X, \mathfrak{B}, \mu, T):=$ $\bigotimes_{n=1}^{\infty}\left(X_{n}, \mathfrak{B}_{n}, \mu_{n}, T_{n}\right)$. If, for each $n \in \mathbb{N}$, there is a function $\alpha_{n}: X_{n} \rightarrow[1,+\infty)$ such that for each $k \in \mathbb{N}$

$$
\alpha_{n}(x)^{-1} \leq \frac{d \mu_{n} \circ T^{k}}{d \mu_{n}}(x) \leq \alpha_{n}(x) \quad \text { at a.e. } \mu_{n} \text {-a.e. } x \in X_{n}
$$

then the dynamical system $(X, \mathfrak{B}, \mu, T)$ is either conservative or totally dissipative. Moreover, if $(Y, \mathfrak{C}, \nu, S)$ is an ergodic conservative nonsingular dynamical system then the direct product $T \times S$ is either conservative or totally dissipative.

Proof. We will prove the second claim only. By the Hopf criterion [DaSi, §2],

$$
\mathcal{D}(T \times S)=\left\{(x, y) \in X \times Y \mid \sum_{k=1}^{\infty} \frac{d(\mu \otimes \nu) \circ(T \times S)^{k}}{d(\mu \otimes \nu)}(x, y)<\infty\right\} .
$$

For each $r>0$, we consider a transformation $\gamma_{r}$ of $X$ by setting $\gamma_{r}\left(x_{1}, x_{2}, \ldots\right):=$ $\left(x_{1}, \ldots, x_{r-1}, T_{r} x_{r}, x_{r+1}, \ldots\right)$. Of course, $\gamma_{r} \in \operatorname{Aut}(X, \mu)$. Denote by $\Gamma$ the transformation group generated by $\gamma_{r}, r \in \mathbb{N}$. It follows from the Kolmogorov 0-1 law 
that $\Gamma$ is ergodic. We claim that $\mathcal{D}(T \times S)$ is invariant under $\gamma_{r} \times I$ for each $r$. Let $(x, y) \in \mathcal{D}(T \times S)$. Since for each $k>0$,

$$
\begin{aligned}
\frac{d \mu \circ T^{k}}{d \mu}\left(\gamma_{r} x\right) & =\frac{d \mu_{r} \circ T_{r}^{k}}{d \mu_{r}}\left(T_{r} x_{r}\right)\left(\frac{d \mu_{r} \circ T_{r}^{k}}{d \mu_{r}}\left(x_{r}\right)\right)^{-1} \prod_{n=1}^{\infty} \frac{d \mu_{n} \circ T_{n}^{k}}{d \mu_{n}}\left(x_{n}\right) \\
& \leq \alpha_{r}\left(T_{r} x_{r}\right) \alpha_{r}\left(x_{r}\right)^{-1} \frac{d \mu \circ T^{k}}{d \mu}(x),
\end{aligned}
$$

it follows that

$$
\begin{aligned}
\sum_{k=1}^{\infty} \frac{d(\mu \otimes \nu) \circ(T \times S)^{k}}{d(\mu \otimes \nu)}\left(\gamma_{r} x, y\right) & =\sum_{k=1}^{\infty} \frac{d \mu \circ T^{k}}{d \mu}\left(\gamma_{r} x\right) \frac{d \nu \circ S^{k}}{d \nu}(y) \\
& \leq \frac{\alpha_{r}\left(T_{r} x_{r}\right)}{\alpha_{r}\left(x_{r}\right)} \sum_{k=1}^{\infty} \frac{d \mu \circ T^{k}}{d \mu}(x) \frac{d \nu \circ S^{k}}{d \nu}(y) \\
& =\frac{\alpha_{r}\left(T_{r} x_{r}\right)}{\alpha_{r}\left(x_{r}\right)} \sum_{k=1}^{\infty} \frac{d(\mu \otimes \nu) \circ(T \times S)^{k}}{d(\mu \otimes \nu)}(x, y)<\infty
\end{aligned}
$$

Thus, $\left(\gamma_{r} x, y\right) \in \mathcal{D}(T \times S)$. Since $\mathcal{D}(T \times S)$ is invariant under $I \times S$, we obtain that $\mathcal{D}(T \times S)$ is invariant under an ergodic transformation group on $X \times Y$ generated by $I \times S$ and $\gamma \times I, \gamma \in \Gamma$. Hence, either $(\mu \otimes \nu)(\mathcal{D}(T \times S))=0$ or $(\mu \otimes \nu)(\mathcal{D}(T \times S))=1$, as desired.

We now apply Proposition 2.6 to IDPFT systems.

Corollary 2.7. Let $\left(X_{n}, \mathfrak{B}_{n}, \mu_{n}, T_{n}\right)$ be an ergodic nonsingular dynamical system on a standard probability space for each $n \in \mathbb{N}$ and let (2-1) hold. Suppose that for each $n \in \mathbb{N}$, there is a $\mu_{n}$-equivalent $T_{n}$-invariant probability measure on $X_{n}$. Let $(X, \mathfrak{B}, \mu, T):=\bigotimes_{n=1}^{\infty}\left(X_{n}, \mathfrak{B}_{n}, \mu_{n}, T_{n}\right)$. Then the dynamical system $(X, \mathfrak{B}, \mu, T)$ is either conservative or totally dissipative. Moreover, if $(Y, \mathfrak{C}, \nu, S)$ is an ergodic conservative nonsingular dynamical system then the direct product $T \times S$ is either conservative or totally dissipative.

Proof. Let $\phi_{n}:=\frac{d \mu_{n}}{d \nu_{n}}$ for each $n>0$. If for each $n>0$, there is a real $\alpha_{n} \geq 1$ such that $\alpha_{n}^{-1} \leq \phi_{n} \leq \alpha_{n}$ almost everywhere then the claim of the corollary follows directly from Proposition 2.6. We now show that the general case can be reduced to the "bounded" one. Indeed, for each $n>0$, we can find a probability measure $\widetilde{\mu}_{n} \sim \mu_{n}$ such that $H^{2}\left(\widetilde{\mu}_{n}, \mu_{n}\right) \leq 2^{-n}$ and the Radon-Nikodym derivative $\frac{d \widetilde{\mu}_{n}}{d \nu_{n}}$ is bounded from above and separated from 0 from below. ${ }^{7}$ Since $\sum_{n=1}^{\infty} H^{2}\left(\widetilde{\mu}_{n}, \mu_{n}\right)<$ $\infty$, it follows from Theorem A that $\mu \sim \tilde{\mu}:=\bigotimes_{n=1}^{\infty} \widetilde{\mu}_{n}$. It remains to note that the conservativeness of a dynamical system does not depend on the choice of quasiinvariant measure within its equivalence class.

2.4. Sharp weak mixing for Maharam extensions of IDPFT systems. We first introduce a "nonsingular analog" of the property ATI (cf. Definition 1.2).

\footnotetext{
${ }^{7}$ For that, take the Radon-Nikodym derivative $\frac{d \mu_{n}}{d \nu_{n}}$ and change it on a subset of very small measure to get the boundedness. The "modified" function will be the Radon-Nikodym derivative $\frac{d \widetilde{\mu}_{n}}{d \mu_{n}}$.
} 
Definition 2.8. A sequence $\left(\xi_{n}\right)_{n=1}^{\infty}$ of probability non-atomic Borel measures on $G$ is called asymptotically translation quasi-invariant (ATQI) if for each $a \in G$ there exists $\zeta_{a}>0$ such that for every $n \in \mathbb{N}$ there are $m>n$ and a Borel subset $W_{n, m} \subset G$ such that

$$
\begin{gathered}
\zeta_{a} \leq\left(\xi_{n} * \xi_{n+1} * \cdots * \xi_{n+m}\right)\left(W_{n, m}\right), \\
\xi_{n} * \xi_{n+1} * \cdots * \xi_{n+m} * \delta_{a} \prec \xi_{n} * \xi_{n+1} * \cdots * \xi_{n+m} \quad \text { and } \\
\zeta_{a} \leq \frac{d\left(\xi_{n} * \xi_{n+1} * \cdots * \xi_{n+m} * \delta_{a}\right)}{d\left(\xi_{n} * \xi_{n+1} * \cdots * \xi_{n+m}\right)}(t) \quad \text { for each } t \in W_{n, m}
\end{gathered}
$$

We will need the following lemma on continuous measures.

Lemma 2.9. Given a standard probability space $(Y, \mathfrak{C}, \nu)$, a nonnegative function $\phi \in L^{1}(Y, \nu)$ such that the measure $\nu \circ \phi^{-1}$ is nonatomic and $\delta \in(0,1)$, then

$$
\max \left\{\int_{A} \phi d \nu \mid \nu(A)=\delta\right\} \geq \frac{\delta}{2} \int_{Y} \phi d \nu .
$$

Proof. Since $\nu \circ \phi^{-1}$ is nonatomic, the function

$$
f:[0,+\infty) \ni t \mapsto f(t):=\nu(\{y \in Y \mid \phi(y)>t\}) \in[0,1]
$$

is continuous and non-increasing. Since $f(0)=1$ and $\lim _{t \rightarrow \infty} f(t)=0$, there is a unique $t_{0}>0$ such that $f\left(t_{0}\right)=\delta$. Let $E:=\left\{y \in Y \mid \phi(y)>t_{0}\right\}$. Then $\max \left\{\int_{A} \phi d \nu \mid \nu(A)=\delta\right\}=\int_{E} \phi d \nu$. Find $n \geq 1$ such that $\frac{1}{n+1}<\delta \leq \frac{1}{n}$. Then there is a partition $Y=Y_{1} \sqcup \cdots \sqcup Y_{n+1}$ of $Y$ into subsets $Y_{k}$ such that $\nu\left(Y_{k}\right)=\delta$ for each $k=1, \ldots, n$ and $\mu\left(Y_{n+1}\right) \leq \delta$. We now have:

$$
\int_{Y} \phi d \nu=\sum_{k=1}^{n+1} \int_{Y_{k}} \phi d \nu \leq(n+1) \int_{E} \phi d \nu \leq \frac{n+1}{n \delta} \int_{E} \phi d \nu \leq \frac{2}{\delta} \int_{E} \phi d \nu .
$$

The next theorem is a nonsingular analogue of Theorem 1.5. The skeleton of the proof is similar to that of Theorem 1.5.

Theorem 2.10. Let a dynamical system $\left(X_{n}, \mathfrak{B}_{n}, \nu_{n}, T_{n}\right)$ be mildly mixing for each $n>0$. Let $\mu_{n}$ be a probability on $X_{n}$ such that $\mu_{n} \sim \nu_{n}$ for each $n \in \mathbb{N}$. Let $\phi_{n}:=\frac{d \mu_{n}}{d \nu_{n}}$ and $(2-2)$ hold. We set

$$
(X, \mathfrak{B}, \nu, T):=\bigotimes_{n \in \mathbb{N}}\left(X_{n}, \mathfrak{B}_{n}, \nu_{n}, T_{n}\right)
$$

and $\mu:=\bigotimes_{n=1}^{\infty} \mu_{n}$. If $T$ is $\mu$-conservative and the sequence of probability measures $\left(\nu_{n} \circ\left(\log \phi_{n}\right)^{-1}\right)_{n=1}^{\infty}$ is ATQI then $T \in \operatorname{Aut}(X, \mu)$ is ergodic of stable type $I I I_{1}$. Moreover, the Maharam extension of $T$ is sharply weak mixing.

Proof. By the Maharam theorem, the Maharam extension $T_{\rho_{\mu}}$ is conservative. Let $C$ be an ergodic conservative transformation of a standard probability space 
$(Z, \mathfrak{Z}, \eta)$. As in the proof of Theorem 1.5 , one can show that $T_{\rho_{\mu}} \times C$ is either totally dissipative or conservative. Suppose that it is conservative and prove that it is ergodic.

Let a function $F \in L^{\infty}(X \times \mathbb{R} \times Z, \mu \otimes \kappa \otimes \eta)$ be invariant under $T_{\rho_{\mu}} \times C$. We first show that $F$ is also invariant under a huge group of transformations. Fix $n>0$. We define a nonsingular automorphism $T^{(n)}$ of $\left(\bigotimes_{k>n} X_{k}, \mu^{(n)}\right)$, where $\mu^{(n)}:=$ $\bigotimes_{k=n+1}^{\infty} \mu_{k}$, and a measure preserving isomorphism $E_{n}$ of $(X \times \mathbb{R} \times Z, \mu \otimes \kappa \otimes \eta)$ onto the product space $\left(X \times \mathbb{R} \times Z,\left(\otimes_{k=1}^{n} \nu_{k}\right) \otimes \mu^{(n)} \otimes \kappa \otimes \eta\right)$ by setting

$$
\begin{aligned}
T^{(n)}\left(x_{k}\right)_{k=n+1}^{\infty} & :=\left(T_{k} x_{k}\right)_{k=n+1}^{\infty} \text { and } \\
E_{n}(x, t, z) & :=\left(x, t+\sum_{k=1}^{n} \log \phi_{k}\left(x_{k}\right), z\right) .
\end{aligned}
$$

Since

$$
\begin{aligned}
T_{\rho_{\mu}}(x, t) & =\left(T_{1} x_{1}, T_{2} x_{2}, \ldots, t+\sum_{k=1}^{\infty} \log \frac{d \mu_{k} \circ T_{k}}{d \mu_{k}}\left(x_{k}\right)\right) \\
& =\left(T_{1} x_{1}, T_{2} x_{2}, \ldots, t+\sum_{k=1}^{\infty}\left(\log \phi_{k}\left(T_{k} x_{k}\right)-\log \phi_{k}\left(x_{k}\right)\right)\right),
\end{aligned}
$$

it follows that

$$
E_{n}\left(T_{\rho_{\mu}} \times C\right) E_{n}^{-1}=\left(T_{1} \times \cdots \times T_{n}\right) \times\left(T^{(n)}\right)_{\rho_{\mu}(n)} \times C .
$$

Since $T_{\rho_{\mu}} \times C$ is conservative, it follows from (2-4) that the product $\left(T^{(n)}\right)_{\rho_{\mu}(n)} \times C$ is also conservative. On the other hand, the function $F \circ E_{n}^{-1}$ is invariant under $E_{n}\left(T_{f} \times C\right) E_{n}^{-1}$. Utilizing these two facts plus the mild mixing of the transformation $T_{1} \times \cdots \times T_{n}$, we deduce from Theorem $\mathrm{B}$ that $F \circ E_{n}^{-1}$ does not depend on the coordinates $x_{1}, \ldots, x_{n}$. Hence, for each transformation $S \in \operatorname{Aut}_{0}\left(X_{1} \times \cdots \times\right.$ $\left.X_{n}, \bigotimes_{k=1}^{n} \nu_{k}\right)$, we have that $F \circ E_{n}^{-1} \circ\left(S \times I \times I_{Z}\right)=F \circ E_{n}^{-1}$. Therefore $F$ is invariant under the transformation $E_{n}^{-1}\left(S \times I \times I_{Z}\right) E_{n} \in \operatorname{Aut}_{0}(X \times \mathbb{R} \times Z, \mu \otimes \kappa \otimes \eta)$ and

$$
E_{n}^{-1}\left(S \times I \times I_{Z}\right) E_{n}=(S \times I)_{\rho_{\mu}} \times I_{Z} .
$$

Denote by $\Gamma$ the group of nonsingular transformations of $(X, \mathfrak{B}, \mu)$ generated by $I \times T_{n} \times I, n \in \mathbb{N}$. Then $\Gamma$ is an ergodic Abelian countable subgroup of $\operatorname{Aut}(X, \mu)$ and $F$ is invariant under $\left\{\gamma_{\rho_{\mu}} \times I_{Z} \mid \gamma \in \Gamma\right\}$ by (2-5).

Claim II. We claim that $\Gamma$ is of type $I I I_{1}$. Equivalently, we will show that each $a \in \mathbb{R}$ is an essential value for the cocycle $\rho_{\mu}$ of $\Gamma$. For that, fix $n>0, \epsilon>0$ and a Borel subset $B \subset X_{1} \times \cdots \times X_{n}$. Denote by $\psi_{k}$ the pushforward of $\nu_{k}$ under $\log \phi_{k}$ for each $k>0$. By ATQI, there is $\zeta_{a}>0$ (which does not depend on $n$ ), $m>n$ and a subset $W_{n+1, m} \subset \mathbb{R}$ such that

$$
\begin{gathered}
\zeta_{a} \leq\left(\psi_{n+1} * \cdots * \psi_{m}\right)\left(W_{n+1, m}\right) \\
\psi_{n+1} * \cdots * \psi_{m} * \delta_{a} \prec \psi_{n+1} * \cdots * \psi_{m} \quad \text { and } \\
\zeta_{a} \leq \frac{d\left(\psi_{n+1} * \cdots * \psi_{n+m} * \delta_{a}\right)}{d\left(\psi_{n+1} * \cdots * \psi_{n+m}\right)}(t) \quad \text { for each } t \in W_{n+1, m} .
\end{gathered}
$$


Choose a countable partition $\mathcal{P}$ of $W_{n+1, m}$ into subsets of diameter no more than $\epsilon$. For each $\Delta \in \mathcal{P}$, we let

$$
\begin{aligned}
& A_{\Delta}:=\left\{y=\left(y_{k}\right)_{k=n+1}^{m} \in X_{n+1} \times \cdots \times X_{m} \mid \sum_{k=n+1}^{m} \log \phi_{k}\left(y_{k}\right) \in \Delta\right\} \quad \text { and } \\
& B_{\Delta}:=\left\{y=\left(y_{k}\right)_{k=n+1}^{m} \in X_{n+1} \times \cdots \times X_{m} \mid a+\sum_{k=n+1}^{m} \log \phi_{k}\left(y_{k}\right) \in \Delta\right\} .
\end{aligned}
$$

Let $\mu_{n+1}^{m}:=\bigotimes_{k=n+1}^{m} \mu_{k}, \nu_{n+1}^{m}:=\bigotimes_{k=n+1}^{m} \nu_{k}$ and $\phi_{n+1}^{m}:=\frac{d \mu_{n+1}^{m}}{d \nu_{n+1}^{m}}$. Dropping off some atoms of $\mathcal{P}$ if necessary, we may assume without loss of generality that $\nu_{n+1}^{m}\left(A_{\Delta}\right)>0$ (and hence $\nu_{n+1}^{m}\left(B_{\Delta}\right)>0$ in view of $(2-6)$ ) for each $\Delta \in \mathcal{P}$. Note that the group $\Gamma_{n+1, m}$ generated by $m-n$ mutually commuting transformations $T_{n+1} \times I \times \cdots \times I, I \times T_{n+2} \times I \times \cdots \times I, \ldots, I \times \cdots \times I \times T_{m} \in$ $\operatorname{Aut}_{0}\left(X_{n+1} \times \cdots \times X_{m}, \nu_{n+1}^{m}\right)$ is ergodic. Suppose that $\nu_{n+1}^{m}\left(A_{\Delta}\right)>\nu_{n+1}^{m}\left(B_{\Delta}\right)$ for some $\Delta \in \mathcal{P}$. We note that for each Borel subset $A^{\prime} \subset A_{\Delta}$,

$$
\mu_{n+1}^{m}\left(A^{\prime}\right)=\int_{A^{\prime}} \nu_{n+1}^{m}\left(A_{\Delta}\right) \phi_{n+1}^{m} d\left(\frac{\nu_{n+1}^{m}}{\nu_{n+1}^{m}\left(A_{\Delta}\right)}\right) .
$$

We now apply Lemma 2.9 to the space $A_{\Delta}$ equipped with the conditional measure $\nu_{n+1}^{m}(\cdot) / \nu_{n+1}^{m}\left(A_{\Delta}\right)$, the function $\nu_{n+1}^{m}\left(A_{\Delta}\right) \phi_{n+1}^{m}$ and $\delta=\nu_{n+1}^{m}\left(B_{\Delta}\right) / \nu_{n+1}^{m}\left(A_{\Delta}\right)$ to obtain a Borel subset $A_{\Delta}^{\prime}$ of conditional measure $\delta$, so

$$
\nu_{n+1}^{m}\left(A_{\Delta}^{\prime}\right)=\nu_{n+1}^{m}\left(B_{\Delta}\right)
$$

and moreover (using (2-7))

$$
\mu_{n+1}^{m}\left(A_{\Delta}^{\prime}\right) \geq \frac{1}{2} \frac{\nu_{n+1}^{m}\left(B_{\Delta}\right)}{\nu_{n+1}^{m}\left(A_{\Delta}\right)} \mu_{n+1}^{m}\left(A_{\Delta}\right) \geq \frac{\zeta_{a}}{2} \mu_{n+1}^{m}\left(A_{\Delta}\right) .
$$

The latter inequality follows from (2-6) because

$$
\frac{\nu_{n+1}^{m}\left(B_{\Delta}\right)}{\nu_{n+1}^{m}\left(A_{\Delta}\right)}=\frac{\left(\psi_{n} * \psi_{n+1} * \cdots * \psi_{n+m} * \delta_{a}\right)(\Delta)}{\left(\psi_{n} * \psi_{n+1} * \cdots * \psi_{n+m}\right)(\Delta)}
$$

By Hopf's lemma, there is a transformation $S_{0} \in\left[\Gamma_{n+1, m}\right]$ such that

- $S_{0} A_{\Delta} \subset B_{\Delta}$ if $\nu_{n+1}^{m}\left(A_{\Delta}\right) \leq \nu_{n+1}^{m}\left(B_{\Delta}\right)$ and

- $S_{0} A_{\Delta}^{\prime} \subset B_{\Delta}$ if $\nu_{n+1}^{m}\left(A_{\Delta}\right)>\nu_{n+1}^{m}\left(B_{\Delta}\right)$.

Let

$$
A^{+}:=\bigsqcup_{\nu_{n+1}^{m}\left(A_{\Delta}\right) \leq \nu_{n+1}^{m}\left(B_{\Delta}\right)} A_{\Delta} \sqcup \bigsqcup_{\nu_{n+1}^{m}\left(A_{\Delta}\right)>\nu_{n+1}^{m}\left(B_{\Delta}\right)} A_{\Delta}^{\prime} \cdot
$$

Then $\mu_{n+1}^{m}\left(A^{+}\right) \geq \frac{\zeta_{a}}{2} \mu_{n+1}^{m}\left(\bigsqcup_{\Delta \in \mathcal{P}} A_{\Delta}\right) \geq \frac{\zeta_{a}^{2}}{2}$. Of course, for each $y \in A^{+}$,

$$
\left(\sum_{k=n+1}^{m} \log \phi_{k}\right)(y)-\left(\sum_{k=n+1}^{m} \log \phi_{k}\right)\left(S_{0} y\right)=a \pm \epsilon .
$$


We now "extend" $S_{0}$ to a transformation $S \in \operatorname{Aut}(X, \mu)$ by setting

$$
S:=I \times S_{0} \times I .
$$

Then $S \in[\Gamma]$ and in view of $(2-8)$,

$$
\rho_{\mu}(S, x)=-a \pm \epsilon \quad \text { whenever } x_{n+1}^{m} \in A^{+} .
$$

We now have that

$$
\left[B \times A^{+}\right]_{1}^{m} \subset[B]_{1}^{n}, \quad S\left[B \times A^{+}\right]_{1}^{m} \subset[B]_{1}^{n}, \quad \mu\left(\left[B \times A^{+}\right]_{1}^{m}\right) \geq \frac{\zeta_{a}^{2}}{2} \mu\left([B]_{1}^{n}\right)
$$

and (2-9) holds for all $x \in\left[B \times A^{+}\right]_{1}^{m}$. Since the set of all cylinders is dense in $\mathfrak{B}$, it follows from Lemma D that $a$ is an essential value of $\alpha$. Thus, Claim II is proved.

The assertion of the theorem follows from Claim II in the very same way as the assertion of Theorem 1.5 follows from Claim I (in the proof of Theorem 1.5).

Remark 2.11. In this remark we clarify some subtle points in the proof of Theorem 2.10. Let $\mathfrak{B}_{0}$ stand for the collection of all cylinders in $X$. Then $\mathfrak{B}_{0}$ is dense in $\mathfrak{B}$ as with respect to $\mu$ as with respect to $\nu$. Though $\mu \perp \nu$, the two measures are equivalent on $\mathfrak{B}_{0}$, i.e. $\mu(B)=0$ if and only if $\nu(B)=0$ whenever $B \in \mathfrak{B}_{0}$. Given a transformation $\theta \in \operatorname{Aut}(X, \mu)$, the Radon-Nikodym derivative $\frac{d \mu \circ \theta}{d \mu}$ is defined up to a subset of zero $\mu$-measure. Hence it has no sense as a function on $(X, \nu)$. However, if we consider transformations of a specific product structure, say $\gamma \in \Gamma$, then $\frac{d \mu \circ \gamma}{d \mu}$ is defined, in fact, up a subset of zero $\mu$-measure from $\mathfrak{B}_{0}$. Therefore, $\frac{d \mu \circ \gamma}{d \mu}$ is well defined as a measurable function on $(X, \nu)$ as well. Thus, the cocycle $\rho_{\mu}: \Gamma \times X \rightarrow \mathbb{R}$ is well defined simultaneously on $(X, \mu)$ and on $(X, \nu)$. Another observation is that given a transformation $S_{0} \in\left[\Gamma_{n+1, m}\right]$, the extension $S:=I \times S_{0} \times I$ of $S_{0}$ to $X$ is a well defined transformation from $\operatorname{Aut}(X, \mu)$ as well as from $\operatorname{Aut}_{0}(X, \nu)$. Thus, though an element of the full group $[\Gamma]$ is defined up to subset of zero measure, "belonging" $S \in[\Gamma]$ is well defined with respect to $\mu$ as well as with respect to $\nu$.

2.5. On type $I I I_{\lambda}$ for rigid IDPFT systems. We would like to emphasize that the conclusion of Theorem 2.10 does not hold if we drop the mild mixing condition on $T_{n}$ and the ATQI property. We illustrate this on a family of IDPFT systems consisting of infinite product of periodic transformations. Let $P=\left\{p_{n} \mid n \in \mathbb{N}\right\}$ be a countable subset of mutually coprime positive integers and $p_{1}<p_{2}<\cdots$. Of course, $p_{n} \rightarrow+\infty$ as $n \rightarrow \infty$. In the examples that we are going to construct in this subsection, $p_{n} \rightarrow+\infty$ very fast (to be specified below). For $n \in \mathbb{N}$, we set $X_{n}:=\left\{0,1, \ldots, p_{n}-1\right\}$ and identify $X_{n}$ with the cyclic group $\mathbb{Z} / p_{n} \mathbb{Z}$. Then $T_{n}: X_{n} \rightarrow X_{n}$, given by $T_{n} x=x+1\left(\bmod p_{n}\right)$, is a bijection of $X_{n}$. The infinite product $T=\bigotimes_{n=1}^{\infty} T_{n}$ is a minimal rotation on the compact totally disconnected Abelian group $X:=\bigotimes_{n=1}^{\infty} X_{n}$. Of course, the Haar measure $\nu$ on $X$ is the only $T$-invariant Borel probability measure on $X$. This measure is the infinite direct product of the equidistributions on $X_{n}, n \in \mathbb{N}$. Fix $\lambda \in(0,1)$. Let

$$
Y_{n}:=\left\{x_{n} \in X_{n} \mid x_{n}<\frac{p_{n}}{2}-\prod_{k=1}^{n-1} p_{k}\right\} \cup\left\{x_{n} \in X_{n} \mid \frac{p_{n}}{2}<x_{n}<p_{n}-\prod_{k=1}^{n-1} p_{k}\right\} .
$$


Let $l_{n}$ be a positive integer such that $l_{n} p_{1} \cdots p_{n-1} \leq p_{n} / 2<\left(l_{n}+1\right) p_{1} \cdots p_{n-1}$. We now set

$$
Z_{n}:=\left\{x_{n} \in X_{n} \mid p_{1} \cdots p_{n-1}<x_{n}<p_{n} / 2\right\}
$$

For each $n>0$, we define a probability measure $\mu_{n}$ on $X_{n}$ by the following conditions:

- $\mu_{n}(j)=\mu_{n}(0)$ for each $j \leq p_{n} / 2$,

- $\mu_{n}(j)=\mu_{n}\left(p_{n}-1\right)$ for each $j>p_{n} / 2$,

$-\frac{\mu_{n}\left(p_{n}-1\right)}{\mu_{n}(0)}=\lambda$.

Of course, such a measure is defined uniquely. It is straightforward to verify that $H^{2}\left(\mu_{n}, \mu_{n} \circ T_{n}^{-1}\right) \rightarrow 0$ as $n \rightarrow \infty$. Passing to a countable subset in $P$, if necessary, we may assume that the following three conditions are satisfied:

(॰) $\sum_{n=1}^{\infty} H^{2}\left(\mu_{n}, \mu_{n} \circ T_{n}^{-1}\right)<\infty$,

(•) $\mu_{n}\left(Y_{n}\right)>1-2^{-n-1}$ for each $n>0$,

(*) $\mu_{n}\left(Z_{n}\right)>\frac{1}{2(\lambda+1)}$ for each $n>0$.

By Theorem A, in view of (o), $T$ is $\mu$-nonsingular ${ }^{8}$, where $\mu=\bigotimes_{n=1}^{\infty} \mu_{n}$.

Proposition 2.12. $(X, \mu, T)$ is of Krieger's type $I I I_{\lambda}$.

Proof. It follows from the Kolmogorov 0-1 law that $T$ is $\mu$-ergodic. We claim that $T$ is of type $I I I_{\lambda}$. Since $\log \frac{d \mu \circ T^{-1}}{d \mu}(x) \in\{n \log \lambda \mid n \in \mathbb{Z}\}$ at a.e. $x \in X$, it suffices to show that $\log \lambda$ is an essential value of the Radon-Nikodym cocycle $\rho_{\mu}$ of $T$. Fix $n>0$ and take a Borel subset $B \subset X_{1} \times \cdots \times X_{n}$. We now set $A:=B \times Z_{n+1} \times Y_{n+2} \times Y_{n+3} \times \cdots \subset X$. Then $A$ is a Borel subset of the cylinder $[B]_{1}^{n}$. Of course, $T^{p_{1} \cdots p_{n}}[B]_{1}^{n}=[B]_{1}^{n}$ and hence $T^{p_{1} \cdots p_{n} l_{n+1}} A \subset[B]_{1}^{n}$. Since

- $T_{m}^{p_{1} \cdots p_{n}}=I$ for each $m=1, \ldots, n$,

$-\frac{d \mu_{n+1} \circ T^{p_{1} \cdots p_{n} l_{n+1}}}{d \mu_{n+1}}\left(x_{n+1}\right)=\lambda$ if $x_{n+1} \in Z_{n+1}$ and

- $\frac{d \mu_{m} \circ T^{k}}{d \mu_{m}}\left(x_{m}\right)=1$ if $x_{m} \in Y_{m}$ and $0 \leq k \leq p_{1} \cdots p_{m-1}$ and every $m>n+1$,

it follows that for each $x=\left(x_{m}\right)_{m=1}^{\infty} \in A$,

$$
\frac{d \mu \circ T^{p_{1} \cdots p_{n} l_{n+1}}}{d \mu}(x)=\prod_{m=1}^{\infty} \frac{d \mu_{m} \circ T_{m}^{p_{1} \cdots p_{n} l_{n+1}}}{d \mu_{m}}\left(x_{m}\right)=\lambda .
$$

We also note that $\mu(A)>\frac{\mu\left([B]_{1}^{n}\right)}{2(\lambda+1)} \prod_{m=1}^{\infty}\left(1-2^{-m-1}\right)$ in view of $(\bullet)$ and $(\star)$. Hence, $\log \lambda$ is an essential value of $\rho_{\mu}$ by Lemma $D$.

\section{Gaussian dynamical systems}

3.1. Integration in Hilbert spaces. Let $\mathcal{H}$ denote a separable infinite dimensional real Hilbert space. Given a Borel probability measure $\mu$ on $\mathcal{H}$, we denote by $\widehat{\mu}$ the characteristic functional of $\mu$, i.e.

$$
\widehat{\mu}(y):=\int_{\mathcal{H}} e^{i\langle x, y\rangle} d \mu(x), \quad y \in \mathcal{H} .
$$

\footnotetext{
${ }^{8}$ Though the topological system $(X, T)$ is a topological odometer, i.e. a minimal rotation on a monothetic compact totally disconnected Abelian group, the nonsingular system $(X, \mu, T)$ should not be confused with the nonsingular product odometers which are well studied in the literature (see, e.g., [HaOs], [DaSi], [Sc]) because $\mu$ does not split into infinite product when $X$ is written in the product form suitable for the odometer "addition with carry".
} 
We note that each Borel probability measure on $\mathcal{H}$ is defined completely by its characteristic functional. If there is a vector $h \in \mathcal{H}$ and a bounded linear operator $B>0$ in $\mathcal{H}$ such that $\widehat{\mu}(y)=e^{i\langle h, y\rangle-\frac{1}{2}\langle B y, y\rangle}$ for all $y \in \mathcal{H}$ then $\mu$ is called the (nondegenerated) Gaussian measure with covariance operator $B$ and mean $h$. Then for each $t \in \mathbb{R}$ and $y \in \mathcal{H}$,

$$
\int_{\mathbb{R}} e^{i t s} d\left(\mu \circ\langle\cdot, y\rangle^{-1}\right)(s)=\int_{\mathcal{H}} e^{i\langle x, t y\rangle} d \mu(x)=e^{i t\langle h, y\rangle-\frac{1}{2} t^{2}\langle B y, y\rangle} .
$$

Therefore the continuous linear functional $\mathcal{H} \ni x \mapsto\langle x, y\rangle$ has normal distribution $\mathcal{N}_{\langle h, y\rangle,\langle B y, y\rangle}$. In particular, each continuous linear functional belong to $L^{2}\left(\mathcal{H}, \mu_{B}\right)$. By the Minlos-Sazonov theorem, $B$ is a nuclear operator, i.e. $\operatorname{tr}(B)<\infty$ [Sk]. Conversely, each strictly positive nuclear operator $B$ in $\mathcal{H}$ determines a unique Gaussian measure on $\mathcal{H}$ with zero mean and covariance operator $B$. We denote this measure by $\mu_{B}$. Thus $\widehat{\mu_{B}}(y)=e^{-\frac{1}{2}\langle B y, y\rangle}$ for all $y \in \mathcal{H}$. We note that $\int_{\mathcal{H}}\langle x, y\rangle d \mu_{B}(y)=0$ for each $h \in \mathcal{H}$. It is well known that

$$
\int_{\mathcal{H}}\langle x, y\rangle\langle z, y\rangle d \mu_{B}(y)=\langle B x, z\rangle
$$

and hence $\operatorname{tr}(B)=\int_{\mathcal{H}}\|y\|^{2} d \mu_{B}(y)$. We now let $\mathcal{H}_{0}:=B^{\frac{1}{2}} \mathcal{H} \subset \mathcal{H}$ and define an inner product and the corresponding norm on $\mathcal{H}_{0}$ by setting

$$
\langle x, y\rangle_{0}:=\left\langle B^{-\frac{1}{2}} x, B^{-\frac{1}{2}} y\right\rangle \text { and }\|x\|_{0}^{2}:=\langle x, x\rangle_{0} \text { for } x, y \in \mathcal{H}_{0} .
$$

Then $\left(\mathcal{H}_{0},\langle., .\rangle_{0}\right)$ is a Hilbert space. We now show that there is a canonical isometric embedding of $\mathcal{H}_{0}$ into $L^{2}\left(\mathcal{H}, \mu_{B}\right)$. For that, we first take $\theta \in B \mathcal{H} \subset \mathcal{H}_{0}$. Then the mapping

$$
l_{\theta}: \mathcal{H} \ni y \mapsto\left\langle B^{-1} \theta, y\right\rangle
$$

is a continuous linear functional on $\mathcal{H}$. Moreover, for all $\theta, \eta \in B \mathcal{H}$,

$$
\left\langle l_{\theta}, l_{\eta}\right\rangle_{L^{2}\left(\mathcal{H}, \mu_{B}\right)}=\int_{\mathcal{H}}\left\langle B^{-1} \theta, y\right\rangle\left\langle B^{-1} \eta, y\right\rangle d \mu_{B}(y)=\left\langle\theta, B^{-1} \eta\right\rangle=\langle\theta, \eta\rangle_{0} .
$$

In particular, the linear mapping

$$
l: B \mathcal{H} \ni \theta \mapsto l_{\theta} \in L^{2}\left(\mathcal{H}, \mu_{B}\right)
$$

is isometric ${ }^{9}$. We note that $B \mathcal{H}$ is dense in $\mathcal{H}_{0}$. Indeed, since the linear span $\mathcal{L}$ of the orthonormal basis in $\mathcal{H}$ consisting of eigenvectors for $B$ is dense in $\mathcal{H}$, it follows that $B^{\frac{1}{2}} \mathcal{L}$ is dense in $\mathcal{H}_{0}$ because $B^{\frac{1}{2}}$ is an isometric isomorphism of $(\mathcal{H},\langle,\rangle$.$) onto$ $\left(\mathcal{H}_{0},\langle, .\rangle_{0}\right)$. It remains to observe that $B^{\frac{1}{2}} \mathcal{L}=B \mathcal{L}=\mathcal{L}$. Since $B \mathcal{H}$ is dense in $\mathcal{H}_{0}$, the isometry $l$ extends by continuity to an isometry from $\mathcal{H}_{0}$ to $L^{2}\left(\mathcal{H}, \mu_{B}\right)$. Thus, for each $y \in \mathcal{H}_{0}$, there is a sequence $\left(\theta_{n}\right)_{n=1}^{\infty}$ of elements from $B \mathcal{H}$ such that $\left\|y-\theta_{n}\right\|_{0} \rightarrow 0$ and the sequence $\left(l_{\theta_{n}}\right)_{n=1}^{\infty}$ converges to some element $l_{y} \in L^{2}\left(\mathcal{H}, \mu_{B}\right)$. Hence a subsequence $\left(l_{\theta_{n_{k}}}\right)_{k=1}^{\infty}$ converges to $l_{y}$ almost everywhere. Let $D_{y}$ denote the set of all $x \in \mathcal{H}$ such that the sequence $\left(l_{\theta_{n_{k}}}(x)\right)_{k=1}^{\infty}$ converges. It is easy to verify that $D_{y}$ is a (Borel) linear subspace of $\mathcal{H}, \mu_{B}\left(D_{y}\right)=1$ and $l_{y}$ is linear on

\footnotetext{
${ }^{9}$ If $B \mathcal{H}$ is furnished with $\|\cdot\|_{0}$.
} 
$D_{y}$. That is why $l_{y}$ is often called a measurable linear functional on $\mathcal{H}$. Moreover, $D_{y} \supset \mathcal{H}_{0}$ and $l_{y}$ is defined uniquely by the restriction to $\mathcal{H}_{0}$ though $\mu_{B}\left(\mathcal{H}_{0}\right)=0$. It is often convenient to write $\left\langle B^{-1} y, x\right\rangle$ instead of $l_{y}(x)$ for $\mu_{B}$-a.a. $x \in \mathcal{H}$. We note that the distribution of $l_{\theta}$ is $\mathcal{N}_{0,\left\langle\theta, B^{-1} \theta\right\rangle}=\mathcal{N}_{0,\|\theta\|_{0}^{2}}$ for each $\theta \in B \mathcal{H}$. Passing to a limit we obtain that the distribution of $l_{y}$ is $\mathcal{N}_{0,\|y\|_{0}^{2}}$ for each $y \in \mathcal{H}_{0}$.

For each $y \in \mathcal{H}$, we denote by $L_{y}$ the rotation by $y$, i.e. $L_{y} x=x+y$ for all $x \in \mathcal{H}$. By the Cameron-Martin theorem (see [Gu, Corollary 7.4], [Sk]),

$$
\begin{gathered}
\mathcal{H}_{0}=\left\{y \in \mathcal{H} \mid \mu_{B} \sim \mu_{B} \circ L_{y}^{-1}\right\} \quad \text { and for each } y \in \mathcal{H}_{0}, \\
\frac{d \mu_{B} \circ L_{y}^{-1}}{d \mu_{B}}(x)=e^{\left\langle B^{-1} y, x\right\rangle-\frac{1}{2}\|y\|_{0}^{2}} \quad \text { at a.e. } x \in \mathcal{H} .
\end{gathered}
$$

3.2. Fock space and exponential map. Given a separable Hilbert space $\mathcal{K}$, the (bosonic) Fock space $\mathcal{F}(\mathcal{K})$ built over $\mathcal{K}$ is the Hilbert space $\bigoplus_{n=0}^{\infty} \mathcal{K}^{\odot n}$. The subspace $\mathcal{K}^{\odot n}$ of $\mathcal{F}(\mathcal{K})$ is called the $n$-chaos in $\mathcal{F}(\mathcal{K}), n \in \mathbb{Z}_{+}$. Given $h \in \mathcal{K}$, we let $\exp _{h}:=\bigoplus_{n=0}^{\infty} \frac{h^{\otimes n}}{\sqrt{n !}} \in \mathcal{F}(\mathcal{K})$. In particular, $\exp _{0}=(1,0,0, \ldots$,$) is called the$ vacuum vector in $\mathcal{F}(\mathcal{H})$. The map $\exp : \mathcal{K} \ni h \mapsto \exp _{h} \in \mathcal{F}(\mathcal{K})$ is called the exponential map. It satisfies the following [Gu]:

(i) exp is continuous,

(ii) $\left\langle\exp _{h}, \exp _{k}\right\rangle_{\mathcal{F}(\mathcal{K})}=e^{\langle h, k\rangle_{\mathcal{K}}}$ for all $h, k \in \mathcal{K}$,

(iii) the set $\left\{\exp _{h} \mid h \in \mathcal{K}\right\}$ is linearly independent and total in $\mathcal{F}(\mathcal{K})$.

Given an orthogonal operator $V$ in $\mathcal{K}$, we can define a linear operator $\exp V$ of $\mathcal{F}(\mathcal{K})$, called the second quantization of $V$, by setting

$$
(\exp V) h^{\otimes n}:=(V h)^{\otimes n} \quad \text { for all } n \geq 0 \text { and } h \in \mathcal{K} .
$$

Then $\exp V$ preserves each chaos in $\mathcal{F}(\mathcal{K})$ and the restriction of $\exp V$ to the first chaos is $V$. Of course, $(\exp V) \exp _{h}=\exp _{V h}$ for each $h \in \mathcal{K}$. The most important property of the Fock spaces is the following one: given a decomposition $\mathcal{K}=\bigoplus_{j=1}^{\infty} \mathcal{K}_{j}$ of $\mathcal{K}$ into an orthogonal sum of subspaces $\mathcal{K}_{j}$, there is a unique unitary isomorphism $\Phi$ of $\left(\mathcal{F}(\mathcal{K}), \exp _{0}\right)$ onto $\bigotimes_{j=1}^{\infty}\left(\mathcal{F}\left(K_{j}\right), \exp _{0}\right)$ such that

$$
\Phi\left(\exp _{\oplus_{j=1}^{\infty} h_{j}}\right)=\bigotimes_{j=1}^{\infty} \Phi\left(\exp _{h_{j}}\right)
$$

for each vector $\bigoplus_{j=1}^{\infty} h_{j} \in \mathcal{K}$ such that $h_{j}=0$ for all but finitely many $j[\mathrm{Gu}$, Proposition 2.3].

Denote the orthogonal group of $\mathcal{K}$ by $\mathcal{O}(\mathcal{K})$. Let $\operatorname{Aff}(\mathcal{K}):=\mathcal{K} \rtimes \mathcal{O}(\mathcal{K})$ stand for the group of affine operators in $\mathcal{K}$. We recall that an operator $A=(f, V) \in \operatorname{Aff}(\mathcal{K})$ acts on $\mathcal{K}$ by the formula $A h:=f+V h$. One can verify that the multiplication law in $\operatorname{Aff}(\mathcal{K})$ is given by:

$$
(f, V)\left(f^{\prime}, V^{\prime}\right):=\left(f+V f^{\prime}, V V^{\prime}\right) .
$$

We note that $\operatorname{Aff}(\mathcal{K})$ is a Polish group if endowed with the product of the norm topology on $\mathcal{K}$ and the weak operator topology on $\mathcal{O}(\mathcal{K})$. We recall the well-known Weyl unitary representation $W=\left(W_{(f, V)}\right)_{(f, V) \in \operatorname{Aff}(\mathcal{K})}$ of $\operatorname{Aff}(\mathcal{K})$ in $\mathcal{F}(\mathcal{K})[\mathrm{Gu}, \S 2.2]$ :

$$
W_{(f, V)} \exp _{h}:=e^{-\langle f, V h\rangle_{\mathcal{K}}-\frac{1}{2}\|f\|_{\mathcal{K}}^{2}} \exp _{f+V h}, \quad h \in \mathcal{K} .
$$


It is well defined due to (ii) and (iii). Of course, $W_{(0, V)}=\exp V$ for each $V \in \mathcal{O}(\mathcal{K})$.

By [Gu, Theorem 7.1], there is a unique (canonical) unitary isomorphism of $L^{2}\left(\mathcal{H}, \mu_{B}\right)$ with $\mathcal{F}\left(\mathcal{H}_{0}\right)$ such that ${ }^{10}$

$$
\exp _{h}(x):=e^{\left\langle B^{-1} h, x\right\rangle-\frac{1}{2}\|h\|_{0}^{2}}, \quad \text { for a.e. } x \in \mathcal{H} .
$$

Moreover, the map $\mathcal{H}_{0} \ni h \mapsto l_{h} \in L^{2}\left(\mathcal{H}, \mu_{B}\right)$ identifies (isometrically) $\mathcal{H}_{0}$ with the first chaos in $L^{2}\left(\mathcal{H}, \mu_{B}\right)$. It follows from (3-1) and (3-3) that

$$
\exp _{h}=\frac{d \mu_{B} \circ L_{h}^{-1}}{d \mu_{B}} \quad \text { for each } h \in \mathcal{H}_{0} .
$$

It is straightforward to verify that the following additional properties for exp hold:

(iv) $\exp _{h}>0$ for each $h \in \mathcal{H}_{0}$,

(v) $\exp _{h} \in \bigcap_{p=1}^{\infty} L^{p}\left(\mathcal{H}, \mu_{B}\right)$ because the map $\mathcal{H} \ni x \mapsto\left\langle B^{-1} h, x\right\rangle-\frac{1}{2}\|h\|_{0}^{2}$ has normal distribution $\mathcal{N}_{-\frac{1}{2}\|h\|_{0}^{2},\|h\|_{0}^{2}}$ and (3-3) holds,

(vi) $\left\|\exp _{h}\right\|_{1}=1$ for each $h \in \mathcal{H}_{0}$,

(vii) the cone $\left\{\sum_{k=1}^{n} a_{k} \exp _{h_{k}} \mid a_{1}, \ldots, a_{n}>0, h_{1}, \ldots, h_{n} \in \mathcal{H}_{0}, n \in \mathbb{N}\right\}$ is dense in the cone $L_{+}^{2}\left(\mathcal{H}, \mu_{B}\right)$ of non-negative functions from $L^{2}\left(\mathcal{H}, \mu_{B}\right)$,

(viii) $\exp _{h} \cdot \exp _{k}=e^{\langle h, k\rangle_{0}} \exp _{h+k}$ for all $h, k \in \mathcal{H}_{0}$ and hence

(ix) $\sqrt{\exp _{h}}=e^{-\frac{1}{8}\|h\|_{0}^{2}} \exp _{h / 2}$ for each $h \in \mathcal{H}_{0}$

(x) $\exp _{h} \circ L_{f}^{-1}=e^{-\left\langle B^{-1} h, f\right\rangle} \exp _{h}=e^{-\langle h, f\rangle_{0}} \exp _{h}$ for all $h, f \in \mathcal{H}_{0}$.

Remark 3.1.

(i) We recall that $\mathcal{H}_{0}$ is determined by the pair $(\mathcal{H}, B)$ (see $\left.\S 3.1\right)$. Conversely, if $\mathcal{H}_{0}$ is given beforehand as an abstract Hilbert space, then it determines uniquely the probability space $\left(\mathcal{H}, \mu_{B}\right)$ for some pair $(\mathcal{H}, B)$ such that $\mathcal{H}_{0}=$ $B^{\frac{1}{2}} \mathcal{H}$. Indeed, if there is another Hilbert space $\mathcal{K}$ and a non-degenerated nuclear operator $C>0$ on $\mathcal{K}$ such that the space $\mathcal{K}_{0}:=C^{\frac{1}{2}} \mathcal{K}$ furnished with the corresponding Hilbert norm is unitarily isomorphic to $\mathcal{H}_{0}$ via some unitary isomorphism $\Psi$ then according to [Gu, Theorem 7.1] and (3-3), there is a unique unitary isomorphism $\Phi$ of $L^{2}\left(\mathcal{H}, \mu_{B}\right)$ with $L^{2}\left(\mathcal{K}, \mu_{C}\right)$ which maps $\exp _{h}$ onto $\exp _{\Psi^{-1} h}$ for each $h \in \mathcal{H}_{0}$. Hence in view of (vii), $\Phi$ maps $L_{+}^{2}\left(\mathcal{K}, \mu_{C}\right)$ onto $L_{+}^{2}\left(\mathcal{K}, \mu_{C}\right)$. Moreover, $\Phi 1=1$. Therefore $\Phi$ is spacial, i.e. there is a measure preserving isomorphism $\theta:\left(\mathcal{H}, \mu_{B}\right) \rightarrow\left(\mathcal{K}, \mu_{C}\right)$ such that $\Phi h=h \circ \theta^{-1}$ for each $h \in \mathcal{H}$.

(ii) Another useful observation is that given a Hilbert space $\mathcal{K}_{0}$, there is another Hilbert space $\mathcal{K} \supset \mathcal{K}_{0}$ and a nuclear operator $C$ of $\mathcal{K}$ such that $C^{\frac{1}{2}}$ is a unitary isomorphism of $\mathcal{K}$ onto $\mathcal{K}_{0}$.

Remark 3.2. Given a decomposition $\mathcal{H}_{0}=\bigoplus_{j=1}^{\infty} \mathcal{H}_{0, j}$ of $\mathcal{H}_{0}$ into an orthogonal sum of subspaces $\mathcal{H}_{0, j}$, consider the corresponding decomposition $\mathcal{H}=\bigoplus_{j=1}^{\infty} \mathcal{H}_{j}$ of $\mathcal{H}$ into an orthogonal sum of subspaces $\mathcal{H}_{j}:=B^{-\frac{1}{2}} \mathcal{H}_{j, 0}, j \in \mathbb{N}$. Let $P_{j}: \mathcal{H} \rightarrow \mathcal{H}_{j}$ denote the orthogonal projection of $\mathcal{H}$ onto $\mathcal{H}_{j}$ and let $B_{j}:=P_{j} B P_{j}^{*}$. Then $B_{j}$ : $H_{j} \rightarrow H_{j}$ is a nuclear operator and $B_{j}^{\frac{1}{2}} \mathcal{H}_{j}=\mathcal{H}_{0, j}$ for each $j \in \mathbb{N}$. Moreover, $\left(\mathcal{H}, \mu_{B}\right)$ splits into the direct product $\left(\mathcal{H}, \mu_{B}\right)=\bigotimes_{j=1}^{\infty}\left(\mathcal{H}_{j}, \mu_{B_{j}}\right)$ of Gaussian probability spaces $\left(\mathcal{H}_{j}, \mu_{B_{j}}\right)$ in such a way that $\left\{\exp _{h} \mid h \in \mathcal{H}_{0, j}\right\}$ is total in $L^{2}\left(H_{j}, \mu_{B_{j}}\right)$ and $\mu_{B_{j}}=\mu_{B} \circ P_{j}^{*}$ for each $j \in \mathbb{N}$.

\footnotetext{
${ }^{10}$ For simplicity sake, we will write $L^{2}\left(\mathcal{H}, \mu_{B}\right)=\mathcal{F}\left(\mathcal{H}_{0}\right)$ and hence identify $\exp _{h}$ with an $L^{2}$-function on $\left(\mathcal{H}, \mu_{B}\right), h \in \mathcal{H}_{0}$.
} 
3.3. Nonsingular Gaussian action of Aff $\mathcal{H}_{0}$. Let $(Y, \mathfrak{C}, \nu)$ be a standard nonatomic probability space. Denote by $\mathcal{U}\left(L^{2}(Y, \nu)\right)$ the group of unitary operators in $L^{2}(Y, \nu)$ and by $\mathcal{U}_{\mathbb{R}}\left(L^{2}(Y, \nu)\right)$ the subgroup of unitaries that preserve the subspace $L_{\mathbb{R}}^{2}(Y, \nu)$ of real valued functions in $L^{2}(Y, \nu)$. Let

$$
U: \operatorname{Aut}(Y, \nu) \ni T \mapsto U_{T} \in \mathcal{U}_{\mathbb{R}}\left(L^{2}(Y, \nu)\right)
$$

stand for the unitary Koopman representation of $\operatorname{Aut}(Y, \nu)$ in $L^{2}(Y, \nu)$. We recall that $U_{T} f:=f \circ T^{-1} \sqrt{\frac{d \mu \circ T^{-1}}{d \mu}}$ for all $f \in L^{2}(Y, \nu)$. The following results are well known:

$(\bullet)\left\{U_{T} \mid T \in \operatorname{Aut}(Y, \nu)\right\}=\left\{V \in \mathcal{U}_{\mathbb{R}}\left(L^{2}(Y, \nu)\right) \mid V L_{+}^{2}(Y, \nu)=L_{+}^{2}(Y, \nu)\right\}$.

(o) $\left\{U_{T} \mid T \in \operatorname{Aut}_{0}(Y, \nu)\right\}=\left\{V \in \mathcal{U}_{\mathbb{R}}\left(L^{2}(Y, \nu)\right) \mid V L_{+}^{2}(Y, \nu)=L_{+}^{2}(Y, \nu), V 1=\right.$ $1\}$.

We also note that $U$ is one-to-one and the image of $U$ is closed in $\mathcal{U}_{\mathbb{R}}\left(L^{2}(Y, \nu)\right)$ in the weak (and the strong) operator topology.

Let $\mathbb{R}^{*}$ denote the multiplicative group of reals. It is straightforward to verify that for each $t \in \mathbb{R}^{*}$, the map $\alpha_{t}: \operatorname{Aff}\left(\mathcal{H}_{0}\right) \rightarrow \operatorname{Aff}\left(\mathcal{H}_{0}\right)$ given by

$$
(f, V) \mapsto \alpha_{t}(f, V):=(t f, V)
$$

is a continuous automorphism of $\operatorname{Aff}\left(\mathcal{H}_{0}\right)$. Moreover, $\alpha_{t_{1}} \alpha_{t_{2}}=\alpha_{t_{1} t_{2}}$ for all $t_{1}, t_{2} \in$ $\mathbb{R}^{*}$.

It is straightforward to verify that for each $A \in \operatorname{Aff}\left(\mathcal{H}_{0}\right)$, the corresponding Weyl unitary operator $W_{A}$ (see (3-2)) preserves the cone

$$
\left\{\sum_{k=1}^{n} a_{k} \exp _{h_{k}} \mid a_{k}>0, h_{k} \in \mathcal{H}_{0}, \text { for each } k=1, \ldots, n \text { and } n \in \mathbb{N}\right\} .
$$

Hence it preserves $L_{+}^{2}\left(\mathcal{H}, \mu_{B}\right)$ in view of (vii) from $\S 3.2$. Therefore by $(\bullet)$, there is a (unique) transformation $T_{A} \in \operatorname{Aut}\left(\mathcal{H}, \mu_{B}\right)$ such that $U_{T_{A}}=W_{\alpha_{1 / 2}(A)}$.

Definition 3.3. $T_{A}$ is called the nonsingular Gaussian transformation generated by $A \in \operatorname{Aff}\left(\mathcal{H}_{0}\right)$.

Since the image of $\operatorname{Aff}\left(\mathcal{H}_{0}\right)$ under the unitary Weyl representation is closed in the unitary group of the space $L^{2}\left(\mathcal{H}, \mu_{B}\right)$ [Gu, Theorem 2.1], it follows that the group $\left\{T_{A} \mid A \in \operatorname{Aff}\left(\mathcal{H}_{0}\right)\right\}$ of nonsingular Gaussian transformations is closed in $\operatorname{Aut}\left(\mathcal{H}, \mu_{B}\right)$.

\section{Proposition 3.4.}

(i) If $V \in \mathcal{O}\left(\mathcal{H}_{0}\right)$ then $T_{(0, V)}$ is the usual (classic) measure preserving Gaussian transformation generated by the orthogonal operator $V$, i.e. $U_{T_{(0, V)}}=\exp V$ (see [LePaTh, Lemma 2]).

(ii) If $f \in \mathcal{H}_{0}$ then $T_{(f, I)}=L_{f}$.

Proof. (i) We note that

$$
U_{T_{(0, V)}} \exp _{h}=W_{(0, V)} \exp _{h}=\exp _{V h}=(\exp V) \exp _{h} .
$$

Hence $U_{T_{(0, V)}}=\exp V$. 
(ii) Using (3-4) and (viii)-(x) from $\S 3.2$ we obtain that

$$
\begin{aligned}
U_{L_{f}} \exp _{h} & =\sqrt{\frac{d \mu_{B} \circ L_{f}^{-1}}{d \mu_{B}}} \exp _{h} \circ L_{f}^{-1} \\
& =\sqrt{\exp _{f}} e^{-\langle f, h\rangle_{0}} \exp _{h} \\
& =e^{-\frac{1}{8}\|f\|_{0}^{2}} \exp _{f / 2} e^{-\langle h, f\rangle_{0}} \exp _{h} \\
& =e^{-\frac{1}{8}\|f\|_{0}^{2}-\langle f, h\rangle_{0}+\frac{1}{2}\langle f, h\rangle_{0}} \exp _{f / 2+h}
\end{aligned}
$$

Hence $U_{L_{f}} \exp _{h}=e^{-\frac{1}{8}\|f\|_{0}^{2}-\frac{1}{2}\langle f, h\rangle_{0}} \exp _{f / 2+h}=W_{(f / 2, I)} \exp _{h}=U_{T_{(f, I)}} \exp _{h}$. It follows that $T_{(f, I)}=L_{f}$.

Corollary 3.5. Every nonsingular Gaussian transformation $T_{(f, V)}$ is the composition of the classic $\mu_{B}$-preserving Gaussian transformation $T_{(0, V)}$ and a $\mu_{B}$ nonsingular translation $L_{f}=T_{(f, I)}$ which is totally dissipative. ${ }^{11}$ These two transformations commute if and only if $V f=f$.

Remark 3.6. Let $(X, \mathfrak{B}, \mu)$ be a standard $\sigma$-finite nonatomic measure space. Let a transformation $S \in \operatorname{Aut}(X, \mu)$ be such that $\sqrt{\frac{d \mu \circ S^{-1}}{d \mu}}-1 \in L^{2}(X, \mu)$. Then a nonsingular Poisson suspension $S_{*}$ of $S$ is well defined on a standard probability space $\left(X^{*}, \mathfrak{B}^{*}, \mu^{*}\right)\left[\right.$ DaKoRo1]. Let $\left.A:=\left(U_{S}, \sqrt{\frac{d \mu \circ S^{-1}}{d \mu}}-1\right)\right) \in \operatorname{Aff}\left(L^{2}(X, \mu)\right)$. It was shown in [DaKoRo1] that $U_{S_{*}}$ is unitarily equivalent to $W_{A}$. It follows that each nonsingular Poisson transformation is unitarily equivalent to a nonsingular Gaussian transformation: $S_{*}$ is unitarily equivalent to $T_{\alpha_{2}(A)}$ (see (3-5)). We do not know if the converse is true even in the classic (finite measure preserving) case.

It is well known that the transformation group $\left\{T_{(f, I)} \mid f \in \mathcal{H}_{0}\right\} \subset \operatorname{Aut}\left(\mathcal{H}, \mu_{B}\right)$ is ergodic (see $[\mathrm{Gu}],[\mathrm{Sk}]$ ). However Krieger's type of it has not been determined so far. We will show that it is type $I I I_{1}$, i.e. a dense countable subgroup of it is of type $I I I_{1}$ (hence every dense countable subgroup is of type $I I I_{1}$ ).

Theorem 3.7. $\left\{T_{(f, I)} \mid f \in \mathcal{H}_{0}\right\}$ is of type $I I I_{1}$.

Proof. Let $\left\{e_{n} \mid n \in \mathbb{N}\right\}$ be an orthonormal basis of $\mathcal{H}$ consisting of the eigenvectors of $B$. Then $B e_{n}=\lambda_{n} e_{n}, \lambda_{n}>0$ for each $n \in \mathbb{N}$ and $\sum_{n=1}^{\infty} \lambda_{n}<\infty$. Denote by $\Gamma$ the group generated by translations $L_{\sqrt{\lambda_{k}} e_{k}}$ for all $k \in \mathbb{N}$. Then $\Gamma$ is an ergodic countable Abelian subgroup of $\operatorname{Aut}\left(\mathcal{H}, \mu_{B}\right)$. We will show that $\Gamma$ is of type $I I I_{1}$.

Denote by $\mathfrak{B}_{n}$ the smallest Borel $\sigma$-algebra on $\mathcal{H}$ such that the map $\mathcal{H} \ni x \mapsto$ $\left\langle x, e_{k}\right\rangle \in \mathbb{R}$ is $\mathfrak{B}_{n}$-measurable for each $k=1, \ldots, n$. Then $\mathfrak{B}_{1} \subset \mathfrak{B}_{2} \subset \cdots$ and the union $\bigcup_{n>0} \mathfrak{B}_{n}$ is dense in $\mathfrak{B}$. We deduce from (3-3) and (3-4) that for each $n>0$,

$$
\log \frac{d \mu_{B} \circ L \sqrt{\lambda_{n+1}} e_{n+1}}{d \mu_{B}}(x)=\frac{\left\langle x, e_{n+1}\right\rangle}{\sqrt{\lambda_{n+1}}}-\frac{1}{2} .
$$

Take $a \in \mathbb{R}$ and $\epsilon>0$. We now let

$$
D_{n}:=\left\{x \in \mathcal{H} \mid a+\frac{1}{2}-\epsilon<\frac{\left\langle x, e_{n+1}\right\rangle}{\sqrt{\lambda_{n+1}}}<a+\frac{1}{2}+\epsilon\right\} .
$$

\footnotetext{
${ }^{11}$ Let $\mathcal{K}$ stand for the orthogonal complement in $\mathcal{H}$ to the 1-dimensional subspace generated by $f$. Then the set $\{s f+k \mid 0 \leq s<1, k \in \mathcal{K}\} \subset \mathcal{H}$ is a Borel fundamental domain for $L_{f}$.
} 
Since $e_{n+1} \perp e_{k}$ for each $k=1, \ldots, n$ and the random variable $\mathcal{H} \ni x \mapsto\left\langle x, e_{k}\right\rangle \in \mathbb{R}$ is Gaussian for all $k=1, \ldots, n+1$ (and the joint distribution is distributions are also Gaussian), it follows that $D_{n}$ is independent of $\mathfrak{B}_{n}$. Moreover, the measure

$$
\begin{aligned}
\mu_{B}\left(D_{n}\right) & =\frac{1}{\sqrt{2 \pi \lambda_{n+1}}} \int_{\left(a+\frac{1}{2}-\epsilon, a+\frac{1}{2}+\epsilon\right) \cdot \sqrt{\lambda_{n+1}}} e^{-\frac{t^{2}}{2 \lambda_{n+1}}} d t \\
& =\frac{1}{\sqrt{2 \pi}} \int_{\left(a+\frac{1}{2}-\epsilon, a+\frac{1}{2}+\epsilon\right)} e^{-\frac{t^{2}}{2}} d t
\end{aligned}
$$

of $D_{n}$ does not depend on $n .{ }^{12}$ We denote it by $\delta>0$. Then for each subset $A \in \mathfrak{B}_{n}$, we have that $L_{e_{n+1}} A=A$ and hence

- $\left(A \cap D_{n}\right) \cup L_{e_{n+1}}\left(A \cap D_{n}\right) \subset A$,

- $\mu_{B}\left(A \cap D_{n}\right)=\mu_{B}(A) \mu_{B}\left(D_{n}\right)=\delta \mu_{B}(A)$ and

- $\log \frac{d \mu_{B} \circ L_{e_{n+1}}}{d \mu_{B}}(x)=a \pm \epsilon$ for each $x \in A \cap D_{n}$.

It follows from Lemma D (see $\S 2.2$ ) that $a$ is an essential value of the logarithm of the Radon-Nikodym cocycle of $\Gamma$. Since $a$ is an arbitrary element of $\mathbb{R}$, the Radon-Nikodym cocycle is ergodic, i.e. $\Gamma$ is of type $I I I_{1}$.

3.4. When nonsingular Gaussian systems are of type $I I_{1}$. We recall a standard definition.

Definition 3.8. Given $V \in \mathcal{O}\left(\mathcal{H}_{0}\right)$, we say that a vector $f \in \mathcal{H}_{0}$ is a $V$-coboundary if there is $a \in \mathcal{H}_{0}$ such that $f=a-V a$.

In this subsection we prove the following statement (cf. [DaKoRo1, Proposition 6.4] and [ArIsMa]).

Theorem 3.9. Let $(f, V) \in \operatorname{Aff}\left(\mathcal{H}_{0}\right)$. For $n \in \mathbb{Z}$, we define $f^{(n)} \in \mathcal{H}_{0}$ by setting $(f, V)^{n}=\left(f^{(n)}, V^{n}\right)$. The following are equivalent:

(i) $T_{(f, V)}$ admits an equivalent invariant probability measure.

(ii) $f$ is a $V$-coboundary.

(iii) The affine operator $(f, V)$ has a fixed point.

(iv) The sequence $\left(f^{(n)}\right)_{n \in \mathbb{Z}}$ is bounded in $\mathcal{H}_{0}$.

Proof. (ii) $\Longleftrightarrow$ (iv) is classic, see [BeKaVa, Proposition 2.2.9], for a proof.

(ii) $\Longleftrightarrow$ (iii) is obvious because the equality $(f, V) a=a$ for some $a \in \mathcal{H}_{0}$ means $f+V a=a$, i.e. $f$ is a $V$-coboundary.

(ii) $\Longrightarrow$ (i) In view of Proposition 3.2,

$$
\frac{d \mu_{B} \circ T_{(f, V)}^{-1}}{d \mu_{B}}=\frac{d\left(\mu_{B} \circ T_{(0, V)}^{-1}\right) \circ T_{(f, I)}^{-1}}{d \mu_{B}}=\frac{d \mu_{B} \circ T_{(f, I)}^{-1}}{d \mu_{B}}=\frac{d \mu_{B} \circ L_{f}^{-1}}{d \mu_{B}} .
$$

Therefore, by (3-4), we obtain that

$$
\frac{d \mu_{B} \circ T_{(f, V)}^{-1}}{d \mu_{B}}=\exp _{f}
$$

\footnotetext{
${ }^{12}$ We use here the fact that the random variable $\left\langle\cdot, e_{n+1}\right\rangle$ has normal distribution $\mathcal{N}_{0, \lambda_{n+1}}$.
} 
Let $f=a-V a$ for some $a \in \mathcal{H}_{0}$. We claim that

$$
\exp _{f}=\frac{\exp _{a}}{\exp _{a} \circ T_{(f, V)}^{-1}} .
$$

Indeed, applying Proposition 3.4 and (viii) and (x) from $\S 3.2$, we obtain that

$$
\begin{aligned}
\exp _{f} \exp _{a} \circ T_{(f, V)}^{-1} & =\exp _{f} \exp _{a} \circ T_{(0, V)^{-1}} \circ L_{f}^{-1} \\
& =\exp _{f}\left((\exp V) \exp _{a}\right) \circ L_{f}^{-1} \\
& =\exp _{f} \exp _{V a} e^{-\langle V a, f\rangle} \\
& =\exp _{f+V a} \\
& =\exp _{a}
\end{aligned}
$$

Since $\exp _{a} \in L^{1}\left(\mathcal{H}, \mu_{B}\right)$, (i) follows from (3-6).

(i) $\Longrightarrow$ (iv) We first note that for each $h \in \mathcal{H}_{0}$,

$$
\|\sqrt{\exp h}\|_{1}=e^{-\frac{\|h\|_{0}^{2}}{8}}
$$

We now have

$$
\begin{aligned}
\left\langle\left(U_{T_{(f, V)}}\right)^{n} 1,1\right\rangle & =\left\langle U_{T_{\left(f(n), V^{n}\right)}} 1,1\right\rangle \\
& =\left\langle\sqrt{\frac{d \mu_{B} \circ T_{\left(f^{(n)}, V^{n}\right)}^{-1}}{d \mu_{B}}}, 1\right\rangle \\
& =\left\|\sqrt{\exp _{f}^{(n)}}\right\|_{1} \\
& =e^{-\frac{\left\|f^{(n)}\right\|_{0}^{2}}{8}} .
\end{aligned}
$$

Suppose that the sequence $\left(f^{(n)}\right)_{n=1}^{\infty}$ is unbounded. Then there is an increasing sequence $n_{1}<n_{2}<\cdots$ such that $\left\|f^{\left(n_{k}\right)}\right\|_{0}^{2} \rightarrow+\infty$ as $k \rightarrow \infty$. Hence $\left\langle\left(U_{T_{(f, V)}}\right)^{n_{k}} 1,1\right\rangle \rightarrow 0$ as $k \rightarrow \infty$. Since the operator $U_{T_{(f, V)}}$ is positive with respect to the cone $L_{+}^{2}\left(\mathcal{H}, \mu_{B}\right)$, it follows that $U_{T_{(f, V)}}^{n_{k}} \rightarrow 0$ weakly as $k \rightarrow \infty$. Since $T_{(f, V)}$ admits an equivalent invariant probability measure, $U_{T_{(f, V)}}$ is unitarily equivalent to the Koopman operator of a probability preserving transformation. The latter does not have subsequences weakly converging to zero because 1 is a fixed point of this operator.

Remark 3.10. In fact, we showed more: if $f=a-V a$ and $\nu$ is a $\mu_{B}$-equivalent $T_{(f, V)}$-invariant measure then $\frac{d \nu}{d \mu_{B}}=\exp _{a}$.

3.5. Gaussian transformations as IDPFT systems. Suppose that we are given an affine operator $(f, V) \in \operatorname{Aff}\left(\mathcal{H}_{0}\right)$. Suppose also that $V$ has no non-trivial invariant vectors. ${ }^{13}$ Using the spectral decomposition of $V$, as in the proof of Theorem 1.6, we can choose an orthogonal decomposition $\mathcal{H}_{0}=\bigoplus_{r=1}^{\infty} \mathcal{H}_{0, r}$ of $\mathcal{H}_{0}$ in such a way that $V \mathcal{H}_{0, r}=\mathcal{H}_{0, r}$ and the orthogonal projection $f_{r}$ of $f$ onto $\mathcal{H}_{0, r}$ is a $V$-coboundary for each $r \in \mathbb{N}$. Let $V_{r}:=V\left\lceil\mathcal{H}_{r}\right.$. Then $\left(f_{r}, V_{r}\right) \in \operatorname{Aff}\left(\mathcal{H}_{0, r}\right)$ for each

\footnotetext{
${ }^{13}$ Equivalently, the measure of maximal spectral type of $V$ has no atom at 1.
} 
$r \in \mathbb{N}$ and $(f, V)=\bigoplus_{r=1}^{\infty}\left(f_{r}, V_{r}\right)$. Let $\mathcal{H}_{r}$ and $\mu_{r}$ stand for the Hilbert space and a Gaussian measure on $\mathcal{H}_{r}$ respectively such that $\mathcal{F}\left(\mathcal{H}_{0, r}\right)$ is canonically isomorphic to $L^{2}\left(\mathcal{H}_{r}, \mu_{r}\right)$ (see Remark 3.1(ii)). Then the standard probability space $\left(\mathcal{H}, \mu_{B}\right)$ is isomorphic to the infinite product $\bigotimes_{r=1}^{\infty}\left(\mathcal{H}_{r}, \mu_{r}\right)$ according to Remark 3.2. It follows that

$$
\left(\mathcal{H}, \mu_{B}, T_{(f, V)}\right)=\bigotimes_{r=1}^{\infty}\left(\mathcal{H}_{r}, \mu_{r}, T_{\left(f_{r}, V_{r}\right)}\right) .
$$

Since $f_{r}$ is a $V_{r}$-coboundary, there is $a_{r} \in \mathcal{H}_{0, r}$ such that $f_{r}=a_{r}-V_{r} a_{r}$ for each $r \in \mathbb{N}$. By Theorem 3.9, the system $\left(\mathcal{H}_{r}, \mu_{r}, T_{\left(f_{r}, V_{r}\right)}\right)$ admits an equivalent invariant probability measure $\nu_{r}$. Moreover, $\frac{d \mu_{r}}{d \nu_{r}}=\exp _{-a_{r}}$ for each $r \in \mathbb{N}$ in view of Remark 3.10. Thus, we have shown that each nonsingular Gaussian dynamical system $\left(\mathcal{H}, \mu_{B}, T_{(f, V)}\right)$ such that $V$ has no non-trivial invariant vectors is IDPFT (see (3-7)). Therefore, Corollary 2.7 yields the following.

Corollary 3.11. If $V$ has no nontrivial invariant vectors then the nonsingular Gaussian dynamical system $\left(\mathcal{H}, \mu_{B}, T_{(f, V)}\right)$ is either conservative or totally dissipative. In fact, if $(Y, \mathfrak{C}, \nu, S)$ is an ergodic conservative nonsingular dynamical system then the direct product $T_{(f, V)} \times S$ is either conservative or totally dissipative.

The following theorem was first proved in [ArIsMa] in the case of mixing $V$. We extend it to the mildly mixing case with a different proof.

Theorem 3.12. Let $T_{(0, V)}$ be mildly mixing and let $f$ not be a $V$-coboundary. If $T_{(f, V)}$ is conservative then the Maharam extension of $T_{(f, V)}$ is sharply weak mixing. In particular, $T_{(f, V)}$ is of type $I I I_{1}$.

Proof. Since $T_{(f, V)}$ is conservative, it follows from (3-7) and Proposition 2.3 that $T_{(f, V)}$ is sharply weak mixing. Let $a_{r}, \mu_{r}$ and $\nu_{r}$ be as above in this subsection. Since $\frac{d \mu_{r}}{d \nu_{r}}=\exp _{-a_{r}}$ for each $r \in \mathbb{N}$, it follows from (3-1) and (3-4) that the distribution $\psi_{r}$ of $\log \frac{d \mu_{r}}{d \nu_{r}}$ defined on $\left(\mathcal{H}_{0, r}, \nu_{r}\right)$ is $\mathcal{N}_{-\left\|a_{r}\right\|_{0}^{2} / 2,\left\|a_{r}\right\|_{0}^{2}}$. Hence, for all $m>n$, we have

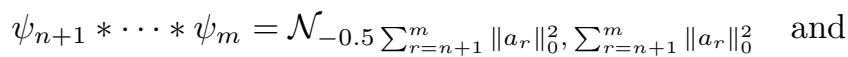

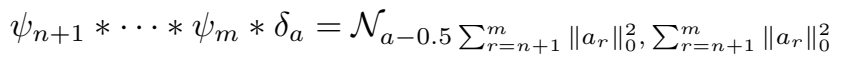

for each $a \in \mathbb{R}$. We are going to show that the sequence $\left(\psi_{r}\right)_{r=1}^{\infty}$ is ATQI. First, it is straightforward to verify that for each $\sigma \in \mathbb{R}$ and $b>0$,

$$
\log \left(\frac{d \mathcal{N}_{b-\sigma^{2} / 2, \sigma^{2}}}{d \mathcal{N}_{-\sigma^{2} / 2, \sigma^{2}}}(t)\right)=\frac{b\left(2 t+\sigma^{2}-b\right)}{2 \sigma^{2}}=\frac{b t}{\sigma^{2}}+\frac{b\left(\sigma^{2}-b\right)}{2 \sigma^{2}}, \quad t \in \mathbb{R} .
$$

Hence, if $t \geq-\sigma^{2}$ and $\sigma^{2} \geq 2 b$ then

$$
\frac{d \mathcal{N}_{b-\sigma^{2} / 2, \sigma^{2}}}{d \mathcal{N}_{-\sigma^{2} / 2, \sigma^{2}}}(t) \geq e^{-b+\frac{b\left(\sigma^{2}-b\right)}{2 \sigma^{2}}}>e^{-\frac{3 b}{4}}
$$

Moreover,

$$
\begin{aligned}
\int_{-\sigma^{2}}^{+\infty} d \mathcal{N}_{-\sigma^{2} / 2, \sigma^{2}}(t) & =\frac{1}{\sigma \sqrt{2 \pi}} \int_{-\sigma^{2}}^{+\infty} e^{-\frac{1}{2}\left(\frac{t+\sigma^{2} / 2}{\sigma}\right)^{2}} d t \\
& =\frac{1}{\sigma \sqrt{2 \pi}} \int_{-\sigma^{2} / 2}^{+\infty} e^{-\frac{t^{2}}{2 \sigma^{2}}} d t \\
& =\frac{1}{\sqrt{2 \pi}} \int_{-\sigma / 2}^{+\infty} e^{-\frac{t^{2}}{2}} d t \\
& 27
\end{aligned}
$$


i.e. $\mathcal{N}_{-\sigma^{2} / 2, \sigma^{2}}\left(\left(-\sigma^{2},+\infty\right)\right)=\mathcal{N}_{0,1}((-\sigma / 2,+\infty))$. Obviously, we have

$$
\psi_{n+1} * \cdots * \psi_{m} * \delta_{a} \sim \psi_{n+1} * \cdots * \psi_{m} \quad \text { for all } n<m .
$$

We now set $\zeta_{a}:=e^{-\frac{3 a}{4}}$. Next, we note that $f$ is not a $V$-coboundary if and only if $\sum_{r=1}^{\infty}\left\|a_{r}\right\|_{0}^{2}=\infty$. Hence for each $n>0$, there is $m>n$ such that $\sum_{r=n+1}^{m}\left\|a_{r}\right\|_{0}^{2}>$ $2 a$. Let $W_{n+1, m}:=\left[-\sum_{r=n+1}^{m}\left\|a_{r}\right\|_{0}^{2},+\infty\right) \subset \mathbb{R}$. Then (3-8) yields that

$$
\frac{d\left(\psi_{n+1} * \cdots * \psi_{m} * \delta_{a}\right)}{d\left(\psi_{n+1} * \cdots * \psi_{m}\right)}(t) \geq \zeta_{a} \quad \text { for all } t \in W_{n+1, m}
$$

Moreover, $\left(\psi_{n+1} * \cdots * \psi_{m}\right)\left(W_{n+1, m}\right)=\mathcal{N}_{0,1}\left(\left(-0.5 \sqrt{\sum_{r=n+1}^{m}\left\|a_{r}\right\|_{0}^{2}},+\infty\right)\right) \approx 1$ if $m$ is large. Hence $\left(\psi_{r}\right)_{r=1}^{\infty}$ is ATQI. It follows now from Theorem 2.10 that the Maharam extension of $T_{(f, V)}$ is sharply weak mixing.

3.6. One-parametric family of nonsingular Gaussian systems. We note that (3-5) determines a one-to-one homomorphism $\mathbb{R}^{*} \ni t \mapsto \alpha_{t}$ from the multiplicative group $\mathbb{R}^{*}$ to the group of continuous automorphisms of $\operatorname{Aff}\left(\mathcal{H}_{0}\right)$. Therefore for each $A \in \operatorname{Aff}\left(\mathcal{H}_{0}\right)$, one can consider a one-parametric family of nonsingular Gaussian transformations $T_{\alpha_{t}(A)} \in \operatorname{Aut}\left(\mathcal{H}, \mu_{B}\right), t \in \mathbb{R}^{* 14}$. Our purpose is this section is to investigate how the dynamical properties of $T_{\alpha_{t}(A)}$ depend on $t$. It is straightforward to verify that the linear operator $-I$ of $\mathcal{H}$ preserves $\mu_{B}$ and conjugates $T_{\alpha_{t}(A)}$ with $T_{\alpha_{-t}(A)}$. Therefore it suffices to consider only the transformations $T_{\alpha_{t}(A)}$ with $t \in \mathbb{R}_{+}^{*}$.

Proposition 3.13 [ArIsMa]. Given $A=(f, V) \in \operatorname{Aff}\left(\mathcal{H}_{0}\right)$ such that $V$ has no non-zero invariant vectors, there is $t_{\mathrm{diss}}(A) \in[0,+\infty]$ such that the transformation $T_{\alpha_{t}(A)}$ is conservative if $0<t<t_{\mathrm{diss}}(A)$ and totally dissipative if $t>t_{\mathrm{diss}}(A)$.

Proof. Let $A=(f, V)$ with $f \in \mathcal{H}_{0}$ and $V \in \mathcal{O}\left(\mathcal{H}_{0}\right)$. It is sufficient to show that if $T_{A}$ is totally dissipative then for each $t>1$, the Gaussian transformation $T_{\alpha_{t}(A)}$ is totally dissipative. Since $T_{A}$ is totally dissipative, the Hopf criterion yields that $\sum_{n=0}^{\infty} \frac{d \mu_{B} \circ T_{A}^{n}}{d \mu_{B}}(x)=\sum_{n=0}^{\infty} e^{\left\langle B^{-1} f^{(n)}, x\right\rangle-\frac{1}{2}\left\|f^{(n)}\right\|_{0}^{2}}<\infty$ for $\mu_{B}$-a.e. $x \in \mathcal{H}$. Hence, there is $N_{x}>0$ such that $\left\langle B^{-1} f^{(n)}, x\right\rangle-\frac{1}{2}\left\|f^{(n)}\right\|_{0}^{2}<0$ for all $n>N_{x}$. It follows that

$$
\left\langle t B^{-1} f^{(n)}, x\right\rangle-\frac{t^{2}\left\|f^{(n)}\right\|_{0}^{2}}{2}<t\left(\left\langle B^{-1} f^{(n)}, x\right\rangle-\frac{\left\|f^{(n)}\right\|_{0}^{2}}{2}\right)<\left\langle B^{-1} f^{(n)}, x\right\rangle-\frac{\left\|f^{(n)}\right\|_{0}^{2}}{2}
$$

for all $n>N_{x}$. Hence $\sum_{n=0}^{\infty} e^{\left\langle t B^{-1} f^{(n)}, x\right\rangle-\frac{1}{2}\left\|t f^{(n)}\right\|_{0}^{2}}<\infty$ for $\mu_{B^{-a}}$ a.e. $x \in \mathcal{H}$. Since $t f^{(n)}=(t f)^{(n)}$, we deduce from the Hopf criterion that $T_{\alpha_{t}(A)}$ is dissipative, as desired.

We recall that the Poincare exponent of $A=(f, V) \in \operatorname{Aff}\left(\mathcal{H}_{0}\right)$ [ArIsMa] is

$$
\delta_{A}:=\inf \left\{\alpha>0 \mid \sum_{n=1}^{\infty} e^{-\alpha\left\|f^{(n)}\right\|_{0}^{2}}<+\infty\right\} \in[0,+\infty]
$$

For completeness of our argument we give a proof of the following proposition.

\footnotetext{
${ }^{14}$ We note that the map $\mathbb{R}^{*} \ni t \mapsto T_{\alpha_{t}(A)}$ is not a group homomorphism
} 
Proposition 3.14 [ArIsMa]. $\sqrt{2 \delta_{A}} \leq t_{\text {diss }}(A) \leq 2 \sqrt{2 \delta_{A}}$.

Proof [ArIsMa]. Let $t>t_{\text {diss }}(A)$. Since $T_{\alpha_{t}(A)}$ is isomorphic to $T_{\alpha_{-t}(A)}$, the two transformations are dissipative. Therefore, by the Hopf criterion,

$$
\sum_{n=0}^{\infty} e^{t\left\langle B^{-1} f^{(n)}, x\right\rangle-\frac{t^{2}}{2}\left\|f^{(n)}\right\|_{0}^{2}}<\infty \quad \text { and } \quad \sum_{n=0}^{\infty} e^{-t\left\langle B^{-1} f^{(n)}, x\right\rangle-\frac{t^{2}}{2}\left\|f^{(n)}\right\|_{0}^{2}}<\infty
$$

at a.e. $x$. Since $e^{t\left\langle B^{-1} f^{(n)}, x\right\rangle}+e^{-t\left\langle B^{-1} f^{(n)}, x\right\rangle} \geq 2$ for each $x \in X$, it follows that $\sum_{n=0}^{\infty} e^{-\frac{t^{2}}{2}\left\|f^{(n)}\right\|_{0}^{2}}<\infty$, i.e. $\delta_{A} \leq t^{2} / 2$ and hence $\delta_{A} \leq t_{\text {diss }}(A)^{2} / 2$.

On the other hand, if $t<t_{\mathrm{diss}}(A)$ then $T_{\alpha_{t}(A)}$ is conservative and hence

$$
\sum_{n=0}^{\infty} e^{t\left\langle B^{-1} f^{(n)}, x\right\rangle-\frac{t^{2}}{2}\left\|f^{(n)}\right\|_{0}^{2}}=+\infty
$$

Therefore,

$$
+\infty=\sum_{n=0}^{\infty} \int_{\mathcal{H}} e^{\frac{1}{2} t\left\langle B^{-1} f^{(n)}, x\right\rangle-\frac{t^{2}}{4}\left\|f^{(n)}\right\|_{0}^{2}} d \mu_{B}(x)=\sum_{n=0}^{\infty} e^{-\frac{t^{2}}{8}\left\|f^{(n)}\right\|_{0}^{2}} .
$$

Hence $\delta_{A} \geq \frac{t^{2}}{8}$ and therefore $\delta_{A} \geq \frac{t_{\mathrm{diss}}(A)^{2}}{8}$.

\section{REFERENCES}

[Aa] J. Aaronson, An introduction to infinite ergodic theory, Mathematical Surveys and Monographs, vol. 50, Amer. Math. Soc, Providence, R. I., 1997.

[AaLiWe] J. Aaronson, M. Lin and B. Weiss, Mixing properties of Markov operators and ergodic transformations, and ergodicity of Cartesian products, Israel J. Math. 33 (1979), 198224.

[AdFrSi] T. Adams, N. Friedman and C. E. Silva, Rank-one weak mixing for nonsingular transformations, Isr. J. Math. 102 (1997), 269-281.

[ArIsMa] Y. Arano, Y. Isono and A. Marrakchi, Ergodic theory of affine isometric actions on Hilbert spaces, preprint arXiv:1911.04272.

[At] G. Atkinson, Recurrence of co-cycles and random walks, J. London Math. Soc. 13 (1976), 486-488.

[Av] N. Avraham-Re'em, On absolutely continuous invariant measures and Krieger-Type of Markov subshifts, preprint, arXiv:2004.05781.

[BeKaVa] B. Bekka, P. de la Harpe and A. Valette, Kazhdan's property (T), New Mathematical Monographs, Volume 11, Cambridge University Press, Cambridge, 2008.

[Da] A. Danilenko, Funny rank-one weak mixing for nonsingular Abelian actions, Isr. J. Math. 121 (2001), 29-54.

[DaKoRo1] A. I. Danilenko, Z. Kosloff and E. Roy, Nonsingular Poisson suspensions, preprint, arXiv:2002.02207.

[DaKoRo2] A. I. Danilenko, Z. Kosloff and E. Roy, Generic nonsingular Poisson suspension is of type $I I I_{1}$, preprint, arXiv:2002.05094.

[DaLe] A. I. Danilenko and M. Lemańczyk, K-property for Maharam extensions of nonsingular Bernoulli and Markov shifts, Ergod. Theory \& Dynam. Sys 39 (2019), 32923321.

[DaSi] A. I. Danilenko, C. E. Silva, Ergodic Theory: Non-singular Transformations, Meyers R. (eds) Mathematics of Complexity and Dynamical Systems, Springer, New York, NY, 2012.

[FuWe] H. Furstenberg and B. Weiss, The finite multipliers of infinite ergodic transformations, Springer Lecture Notes in Math., vol. 688, pp. 127-132. 
[Gu] A. Guichardet, Symmetric Hilbert spaces and related topics, Springer Lecture Notes in Mathematics, vol. 261, 1972.

[HaOs] T. Hamachi and M. Osikawa, Ergodic groups of automorphisms and Krieger's theorems, Seminar on Mathematical Science of Keio Univ. 3 (1981), 1-113.

[Ka] S. Kakutani, On equivalence of infinite product measures, Ann. Math 49 (1948), 214-224.

[KoSo] Z. Kosloff and T. Soo, The orbital equivalence of Bernoulli actions and their Sinai factors, preprint, arXiv:2005.02812.

[LeLeSk] M. Lemańczyk, E. Lesigne and D. Skrenty, Multiplicative Gaussian cocycles, Aequationes Math. 61 (2001), 162-178.

[LePaTh] M. Lemańczyk, F. Parreau, J.-P. Thouvenot, Gaussian automorphisms whose ergodic self-joinings are Gaussian, Fundamenta Math. 164 (2000), 253-293.

[MaVa] A. Marrakchi and S. Vaes, Nonsingular Gaussian actions: beyond the mixing case, preprint, arXiv:2006.07238.

[Ni] M. S. Nikulin, Hellinger distance, Encyclopedia of Mathematics, (M. Hazewinkel, eds.), ISBN 978-1-55608-010-4, Springer Science+Business Media B.V. / Kluwer Academic Publishers.

[Sc] K. Schmidt, Cocycles of ergodic transformation groups, Lecture Notes in Mathematics, vol. 1, MacMillan, India, 1977.

[SiTh] C. E. Silva and P. Thieullen, A skew product entropy for nonsingular transformations, J. London Math. Soc. 52 (1995), 497-516.

[Sk] A. V. Skorohod, Integration in Hilbert spaces, Springer-Verlag, Berlin, Heidelberg, New York, 1974.

B. I. Verkin Institute for Low Temperature Physics \& Engineering of National Academy of Sciences of Ukraine, 47 Nauky Ave., Kharkiv, 61103, UKRAine

E-mail address: alexandre.danilenko@gmail.com

Faculty of Mathematics and Computer Science, Nicolaus Copernicus University, ul. Chopina 12/18, 87-100 Toruń, Poland

E-mail address: mlem@mat.umk.pl 